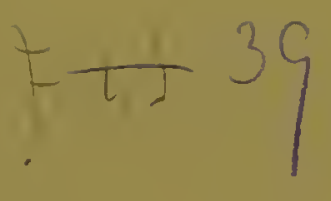

\title{
Zur Dioptrik und Ophthalmoskopie der Fisch- und Amphibienaugen.
}

Von

\author{
J. Hirschberg.
}

In meiner kurzen Mittheilung zur rergleichenden Ophthalmoskopie ${ }^{1}$ musste ich darauf hinweisen, dass noch weitere Untersuchungen nöthig sein werden, um den Gegenstand auch nur zu einem vorläufigen Abschluss zu bringen.

Zunächst wollte ich mir erlauben, einige kurze Bemerkungen über das physikalisch-dioptrische Terhalten der Fisch- und Amphibienaugen, sowie einige Skizzen ihres Augengrundbildes anzuschliessen.

So überwältigend gross und für uns vorwaltend mit der Praxis beschäftigte Aerzte kaum übersehbar auch das vergleichend anatomische IIaterial ist, welches zahlreiche Forscher hinsichtlich der Structur des Auges im Thierreiche angesammelt haben: so spärlich sind die Untersuchungen das physikalischen Verhaltens lebender Fisch- und Amphibienaugen.

Nichtsdestoweniger ist der so naheliegende Unterschied in den dioptrischen Terhältnissen des Wirbelthierauges, je nachdem es sich um Luftr,ler um Wasserbewohner handelt, den Physiologen seit langer Zeit gelāufig und um so leichter aufgefunden worden, als schon der grobe Vergleich eines Fisch- und eines etwa gleich grossen Landwirbelthierauges ganz f,emerkenswerthe Differenzen in der äusseren Gestaltung erkemnen lïsst. In rlam Handbuch der Physiologie von Johannes Müller, das noch heute, nahezu ein hallses Jalırhundert nach seinem Erscheinen, eine so wichtige Fundgrul,e werthwoller Daten darstrilt, heisst es (1837, II, s.314) folgendermaasien: „Yei den in Wasser lebenden Thieren ist die Linse immer con-

'Ties Archiv. 1852. S. 82. 
vexer, als bei den in Luft lebenden, bei den Fischen ist sie kugelrund, bei den Sepien sogar länglich in der Richtung der Achse. Dagegen ist die Hornhaut der in Wasser lebenden Thiere viel flacher als bei den in Luft lebenden. Diesen Thieren würde eine convexe Hornhaut von keinem Nutzen scin, indem die wässrige Feuchtigkeit durch Brechkraft sich wenig ron dem äusseren Wasser, worin die Thiere leben, unterscheidet; während die Brechung durch die Cornea und wässrige Feuchtigkeit bei den in Juft lebenden Thieren sehr gross ist. Dagegen muss die Brechung bei den in Wasser lebenden Thieren durch die stärkere Conrexität der Linse ersetzt werden. Die Linse der Fische ragt mit der vorderen Hälfte durch die Pupille in die vordere Augenkammer."

Diese Darstellung ist durchaus einwandsfrei und bedarf auch keiner Erläuterung, während ich bezüglich einer der neueren Arbeiten auf diesen Gebiete mehrere Bemerkungen zu machen genöthigt bin. Ich meine die Monographie des Hrn. Dr. Felix Plateau (d. J.) über das Sehen der Amphibien und Fische, welche am 2. Juli 1866 der königl. Akademie ron Belgien vorgelegt und von derselben preisgekrönt worden ist. (Vgl. Acad. Royale de Belgique, Extr. du 'Tome XXIII des Mémoires couronnés.)

Der Hr. Verfasser glaubt durch Beobachtung, Theorie und Experiment die folgenden Sätze begründet zu haben:

1) Chez tous les poissons la portion médiane de la cornée est aplatie, de sorte que le rapport entre le rayon de courbure de cette portion et l'axe de l'oeil est beaucoup plus grand que chez les animaux terrestres; cet aplatissement existe même chez ceux des poissons auxquels on a attribué la cornée la plus convexe. - 2) En conséquence de cette conformation et de la sphéricité de leur crystallin, les poissons voient dans l'air aussi bien que dans l'eau; seulement leur distance de vision distincte est un peu plus grande dans ce dernier milieu. - 3) L'oeil des amphibies a une structure identique ou très analogue à celle de l'oeil des poissons, d'où il résulte nécessairement que les amphibies possèdent également la faculté de roir avec netteté dans l'air et dans l'eau, et à peu près à la même distance, sans que, pour passer d'un milieu à l'autre, ils doivent mettre en jeu leur pouvoir d'accommodation.

Hr. Plateau giebt an, dass er zuerst die Frage von dem Sehen der Fische und Amphibien experimentell in Angriff genommen habe.

Unleugbar ist dies sein Verdienst. Ja, seine Arbeit scheint die einzige zu sein, welche dieses Gebiet umfasst, und ist bisher als miassgebend betrachtet worden.

Auch Hr. Prof. Leuckart hat in seiner vortrefflichen Organologie des Auges, die 1876 in dem Graefe-Saemisch'schen Handbuch der Augenhrilkunde erschienen ist und den heutigen Standpunkt unserer Kenntnisse 
ebeuso bündig wie erschöpfend zusammenfasst, den Standpunkt von Platean ohue Vorbehalt angenommen.

\section{Es heisst bei Leuckart Seite 155:}

..Dass auch das Brechungsrermögen des umgehenden Mediums auf die Bildung der optischen Apparate influirt, ist bei Erwähnung der Wasserthiere schon angedentet. Wir werden bei einer späteren Gelegenheit noch weiter darauf zurückiommen und fügen hier nur die Bemerkung bei, dass das Auge der amphibiotischen Thiere, derjenigen also, die so gut im Wasser wie in der Luft leben und sehen, den Bau der Wasserthiere wiederholt (Plateau.) Bei beiden ist es ausschliesslich die Linse, die das Retinalbild erzeugt. Die Cornea, die sonst in der Luft auf den Gang der Lichtstrahlen einen erheblichen Einfluss ausübt, ist durch ibre platte Form aus der Reihe der brechenden Medien ausgeschlossen, sodass die betrefienden Thicre, auch oline besondere Adaptationsleistungen, in der Luft so gut wie im Wasser zu seheu rermögen. Nur insofern besteht einiger Cuterschied, als das deutliche Sehen in der Luft - der in beiden Uedien rerschiedenen Focalmeite entsprechend - erst in etwas grösserer Entfernung ron dem duge anhebt."

Es wäre also nach Platea und Leuckart, renn wir ron eimer accommodativen Terändorung der Krystalllinse absehen, das S'ch ema des

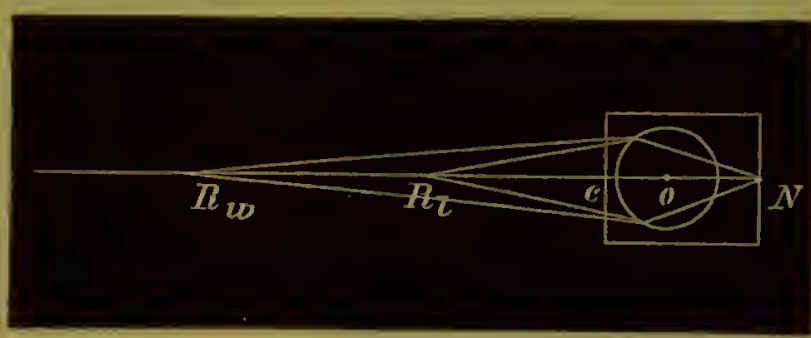

Fig. 1.

Schema des Strahlenganges im Fisch- (nnd Amphibien-) Auge nach F. Platean.

$R w$ Fernpunkt in Wasser.

$R l$ Fernpunkt in Luft.

Die Strecke $R w R l$ ist relativ grösser gezeichnet, als die Zahlen Plateau's zulassen. Das von einem Netzhautpunkt ausgehende Strahlenbündel ist im Kammerwasser stark convergent.

Fisch - und Amphibienauges das folgende (rgl. Fig. 1): Ein geschlossener Kasten sei rorn ron einer plauparallelen Glasplatte $(c)$ begrenzt, der Hohlraum sei mit Wasser gefüllt und entlaalte die kugelige Krystalllinse von stärkerem Brechungsvermögen, sowie linten (dicht hinter dem hinterell I.insenprsl) einen lichtauffangenden Schirm $(N): R w$ sei der Punkt, für den do. A pparat eingerichtet ist, wenn er in Wasser; $R l$, wenn er sich in Luft befindet.

Von der Wirkung, welche eine ebene Trennungsfl̈che zwischen zwei sptisch verschiedenen Herlien auf den Gaug eines schmalen homocentrischen Lichtstrahlenbündels ausübt, kanı man leicht $\Lambda$ ufschluss erlalten, wenn 
in der für eine kugelig e Trennungsfäche einfach abzuleitenden Gleichung ${ }^{1}$

$$
\text { (1) } \frac{n_{1}}{f_{1}}+\frac{n_{2}}{f_{2}}=\frac{n_{2}-n_{1}}{r}
$$

(in der $n_{1}$ den Brechungsindex des ersten, $n_{2}$ den des zweiten Mediums, $f_{1}$ Objectdistanz, $f_{2}$ Bilddistanz, $r$ den Krümmungsradius bedeutet) $r$ unendlich gross gesetzt wird, so dass das Glied

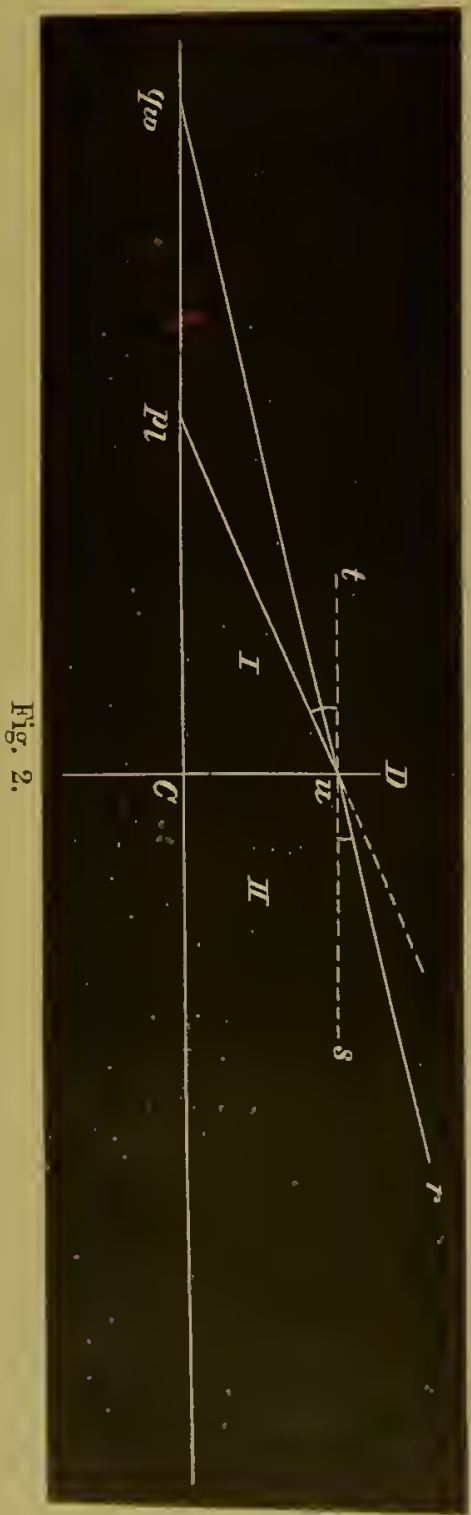
$\frac{n_{2}-n_{1}}{r}$ verschwindet: es folgt dann

(2) $f_{2}=-\frac{n_{2}}{n_{1}} f_{1}$

oder für den Uebergang aus Luft in Wašer $f_{2}=-\frac{4}{3} \cdot f_{1}$. Darnach ist $f_{3}=\infty$, w nn $f_{1}=\infty$.

Ein paralleles Strahlenbündel wird nicht abgelenkt. Stellt aber $p l$ (Fig. 2) einen in Luft $(I)$ befindlichen, in endlicher ...ntfernung von $C$ belegenen Objectpunkt dar, so liegt das Bild, das die ebene Trenn agsflache $C D$ beim Uebergang in Wasser von $p l$ entwirft, weiter ab von $C$ als das ( jject, im Verhältniss von $4: 3$, und auf der āmlichen Seite der Trennungsfläche, also in qw. Convergirt ein aus Wasser $(I I)$ austretendes Strahlenbündel nach $q w$, so wird beim Uebergang in Luft das Bündel in $p l$ vereinigt. Nach dem Brechungsgesetz muss ja der Winkel, welchen ein Lichtstrahl mit dem Einfallsloth bildet, in Luft immer grösser sein, als in Wasser.

$$
(\angle t u p>\angle r u s ; \angle r u s<\angle t u p \text {.) }
$$

Wenn wir also das erwähnte Schema innehmen wollten, so hätten wir bei Leuckart einen kleinen Druckfehler zu velbessern und jedcnfalls mit Pla'eau zu setzen: „Das Sehen in Luft heb, in geringerer Entfernung vom Auge an, als im Wasser". Aber kaun denn überhaupt die Vorderfläche der Fischlornhaut in dioptrischem Sinne als eine Ebcue betrachtet werden? Stimmt der dioptrische Bau des Amphibienauges mit dem des Fïschauges überein? Beide Fragen müssen ' Vgl. den Anhang. 
ebensoviel wie die der menschlichen Krystalllinse beträgt: es räre dies weniger als nach Leuckart, aber doch nicht Null. $\left(\frac{1}{8}+\frac{1}{60}=\frac{1}{7}\right)$. Sähe das Hechtuuge in Wasser auf grössere Distanzen, so hätte es doch noch in Luft cine starke Kurzsichtigkeit, nämlich einen Fermpunktsabstand von $60 \mathrm{~mm}$ oder nahezu 21/". Somit widersprechen Platea u's cigene '/ahlen auf das bestimmteste seinen Annahmen über das Sehen der Fische.

Aber ror Allem ist es geboten, diese Zahlen cincr experimentellen Nachprüfung am lebenden Thiere zu unterziehen. Nan prüft, wem man richtige Resultate erlangen will, die Grösse des Reflexbildes, welches die Mitte der Hornhaut des lebenden Thieres von einem gegebenen Gegenstande entrirft.

Es ist dies das feinste Tasten, mit dem Lichtstrahl. Helmholtz's Ophthalmometer braucht man natürlich für solche approximative Bestimmungen nicht. Man misst die Breite des Reflexbildes, welches die Hornhant ron einer Fensteröffnung entwirft. (Ich wählte eine Objectbreite ron 1000, eine Objectferne ron $3500 \mathrm{~mm}$.) Wird grössere Genauigkeit gewünscht, so kann das von mir am 14. Juli 1876 der Berliner physiologisehen Gesellschaft mitgetheilte Verfahren in Anwendung gezogen werden: „Um die Messung des Hornhantkrümmungsradius zu einer bequemen und schnell ausführbaren Methode der Praxis zu machen, werden in der Höbe des zu untersuchenden Auges zwei Lichtflammen aufgestellt, so dass ihre gegenseitige Entfermung 1 Meter beträgt und das zu untersuchende Auge rom Mittelpunkt ihrer Halbirungslinie 1 Meter entfernt ist. Mit dem Pupillometer misst man $\beta$, die Distanz der beiden Lichtflammen im Spiegelbildchen der Hornhaut, dann ist direct $\beta$ die Brennweite oder der halbe Krümmungsradius des Hornhautsspiegels. Denn, ist $b$ ein Object, $\beta$ sein Bild, a der Abstand des relativ fernen Objects rom Krümmungsmitteljunkt des Convexspiegels, so ist $\frac{\beta}{b}=\frac{\frac{1}{2} r}{a}$, folglich, da wir $a=b$ gemacht haben. $\frac{1}{2} r=\beta . “ 1$

Sehr bequem ist auch zur Hornhautmessung das Keratoskop rou Placido, ${ }^{2}$ eine schiessscheibenähnliche Figur mit centraler Durchbohrung. Ich habe das Tnstrument stabil gemacht und mit einer Millimeterscala versehen, die durch ein kleines Planspiegelehen nach dem scheinbaren ort des Hornlautspiegelbildes hin reflectirt wird. Ist die Scheibe 8 Zoll breit und 8 Zoll entfernt ron der Hornhaut, so hat man nur die Zahl der

Vergl. in diesem Archiv, 1878. S. 581. 582.

2 Vgl. Centralblall für Augenheilkunde. 1882. S. 30 u. fi. 
Theilstriche, welche der Breite des Hornhautreflexbildes entspricht, mit 2 zu multipliciren, um $r$ zu finden. Gleichzeitig gewinnen wir mit einem Blick eine unmittelbare Anschauung von der Wölbung des Hornhautspiegels und der etrraigen Unregelmässigkeiten desselben, sowohl bei Thieren als auch bei Menschen.

Ich habe auf solche Weise verschiedene Fische und Frösche untersucht und will mich begnügen, einige Resultate der Beobachtungen mitzutheilen.

Ein grosser Hecht ron $60 \mathrm{~cm}$ Länge und 1 Kilo Gewicht zeigte folgende Mraasse:

Rechtes Auge. Linkes Auge.

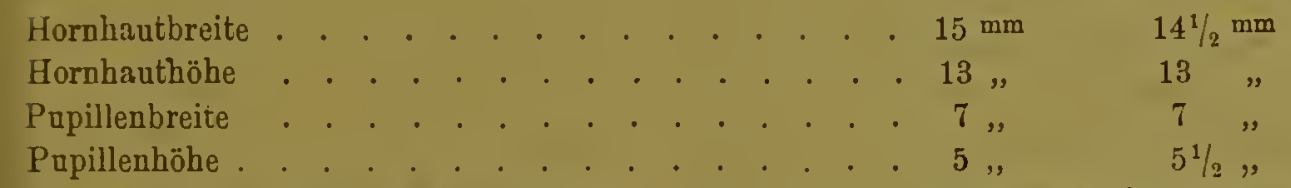

Fensterreflesbild inmitten der Pupille . . . . . . . $1.75 \mathrm{~mm}$ breit $1.75 \mathrm{~mm}$ breit näher dem nasalen Rande der Pupille. am nasalen Rande der Pupille . . . jenseits des nasalen Pupillenrandes,

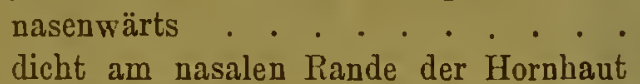
nahe dem temporalen Rande der Pupille im Pupillargebiet . . . . . . . . am temporalen Rande der Pupille .

jenseits desselben, schläfenwärts . . . am Schläfenrande der Cornea . . . . $2 \cdot 5$ " $\quad 2.5$ " " vor der Mitte des oberen Pupillarrandes $2 \cdot 5 \quad, \quad$ " $2.5 \quad$, " weiter oberhalb des ob. Pupillarrandes 3.5 ", ", $\begin{array}{lllllll}\text { vor der Mitte des unteren Pupillarrandes } 2 \cdot 5, & , & 2 \cdot 5 & , & & & \\ \text { weiter nnterhalb des ob. Pupillarrandes } & & & 3 \cdot 5 & \end{array}$

Am kleinsten und regelmässigsten war das Bild im nasalen Drittel des Pupillarbereiches.

Jehr als die laterale Hälfte des Pupillarbereiches der Hornhaut ist stark abgeflacht, ebenso eine schmale extrapupillare Zone der umgebenden Hr,rnhaut.

Fs ist nämlich $\frac{1000}{3500}=\frac{b}{\frac{r}{2}}$, wo $b$ die Breite des Reflexbildes.

Hieraus folgt:

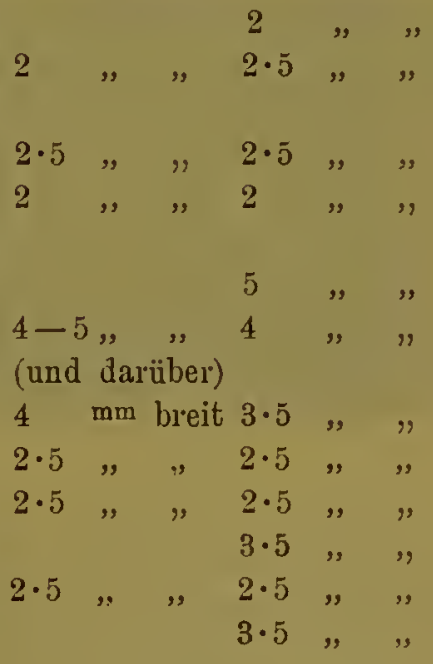

$$
\begin{aligned}
& \frac{r}{2}=3 \cdot 5 \mathrm{~mm}, r=7 \mathrm{~mm} \text { für } b=1 \mathrm{~mm} \\
& \frac{r}{2}=6.2, \quad r=12 \cdot 4, \quad, \quad, \quad b=1 \cdot 7.5 \mathrm{~mm} \\
& \frac{r}{2}=7 \quad, \quad r=14 \quad, \quad, b=2 \mathrm{~mm}
\end{aligned}
$$




$$
\begin{aligned}
& \frac{r}{2}=8 \cdot 75^{\mathrm{mm}}, r=17 \cdot 5^{\mathrm{mm}} \text { für } b=2 \cdot 5 \mathrm{~mm} \\
& \frac{r}{2}=10 \cdot 5, \quad r=21 \quad " \quad, b=3 \quad \text { " } \\
& \frac{r}{2}=12 \cdot 25, r=\underline{25} \quad " \quad b=3 \cdot 5, \\
& \frac{r}{2}=14 \quad " \underline{r=28} \quad " \quad, b=4 \quad " \\
& \frac{r}{2}=17.5, \quad \underline{r=35} \quad " \quad, l=5 \quad "
\end{aligned}
$$

Nicht bloss $20 \mathrm{~mm}$, wie Platea u annimmt, sondern sogar $28 \mathrm{~mm}$ lang wird der Hornhaut-Krümmungsiadius $r$ innerhalb des Pupillargebietes grosser Hechtaugen, und zwar mehr schläfenwärts; aber inmitten des Pupillargebietes und nasenwïrts ist $r$ nur 12 bis $14^{\mathrm{mm}}$, d. h. 1.5 bis 2 mal

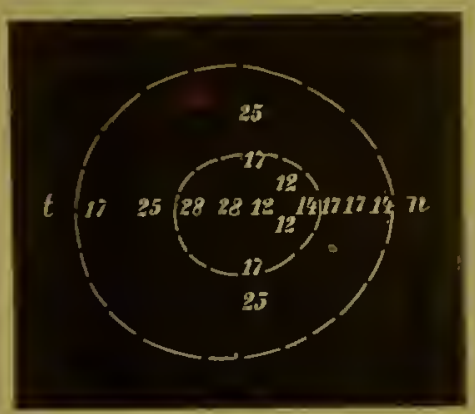

Fig. 3.

Hechteornea in doppelter Grösse; die Zahlen bedeuten die Krümmungsradien der einzelnen Stellen, $n$ nasaler Rand der Cornea, $t$ temporaler. so gross, als der Krümmungsradius der menschlichen Hornhaut. Ton einem einzelnen Krümmungşradius der Hechtcornea, soweit sie optisch in Betracht liommt, kann man gar nicht reden: das lehrt ein Blick auf die schematische Fig. 3, welche die Hechtcornea (in doppelter Tergrösserung) mit den eingezeichneten wirkichen (horiz.) Kírümmungsradien der einzelnen Stelleı enthält.

Theoretisch wird schwer zu entscheiden sein, welcher Krrümmungsradius bei der Lichtbrechung aus Luft in das Fischauge hauptsächlich in Betracht kommt. Entse heidend ist der. Versuch. Der lebende Hecht wird in Luft gehalten, man beleuchtet mit dem Augenspiegel die Pupille. Die brechenden Medien des Fischauges selbst entwerfen ein umgekehrtes ${ }^{1}$ Bild der Netzhaut und zwar des Selnerveneintritts. Ich messe die maximale Entfernung $D$ zwischen meinem und dem Fischauge, bei der ich jenes Bild noch am schärlsten sehe und finde sie gleich $12^{1} / 2$ bis 13 Zoll; meine fernpunktsdistanz $d$ beträgt 10"; folglich liat das Hechtauge in Luft eine excessive Kurzsichtigkeit mit einem Fermpunktsabstand $R=D-d$ $=2^{1} / 2$ bis $3^{\prime \prime}$. Das umgekehrte Netahautbild ist allerdings starli astigmatisch. Ein möglicher Fehler voll etwa 1/2" ist gern zugegeben.

${ }^{1}$ Dass das Netzhautbild ein umgekchrtes reelles Iuftbild ist, kann man an scineu optiseben Eigenschaften leieht erkennen; es versehiebt sich nach reehts, wenn der Kopf des Beobachters nach links geht; es versehwindet, wenn der Beobachter sein Auge an das fremde unmittelbar heranbringt. 
Jetzt gilt es, die Refraction lessclben lebenden Hechtauges unter II asser zu bestimmen. Dies ist schwierig, aber doch mit Sicherheit ausführbar. Ich bedecke den pupillaren Hornhautbereich mit Wasser und lege darauf ein Stückchen ron einem Deckgläschen für mikroskopische Präparate. Die Horuhaut ist jetzt in Wasser getaucht, ihre Wirkung null; die brechende Trirkung des planparallelen Deckgläschens ist gleichfalls null. Ich finde nummehr mit Hilfe des aufrechten Netzhautbildes objectiv eine Myopie des in Wasser getauchten Fischauges von etwa 24 Zoll ${ }^{1}$ Fernpunktsabstand $(=1 \cdot 5 D)$, also einen ganz schwachen Grad. Mit Ueberraschung sehe ich, wie ausserordentlich viel besser der optische Apparat dieses Fischauges in Wasser als in Luft arbeitet. Ich erhalte ein regelmässiges, natūrlich stark rergrōssertes Bild der Netzhautmitte: feinste Sehnerrenfasern ziehen radiär in mehr horizontalen Richtungen über den röthlichen Augengrund; dahinter liegt ein System von feinen senkrechten Linien, das ganze ist wie chagrinirt durch glitzernde Punkte.

Das umgekehrte Bild des Sehnerreneintritts, mit der Linse $+2^{\prime \prime}$ entworfen, ist jetzt (bei aufgelegtem Deckglas) etwa dreimal breiter als rorher, wenn das Auge in Luft sich befand: natürlich, je schwächer die Myopie, desto stärher die Tergrösserung des umgekehrten Bildes. Taucht das Hechtauge aus Wasser empor in Luft, so fügt es sich durch Inkrafttreten der Hornhautbrechung eine Linse zu, deren Brechkraft also in Zollmaass gleich

$$
\frac{1}{2 \frac{1}{2}}-\frac{1}{24}=\frac{1}{2 \cdot 8} "
$$

2. S Zoll sind etwa $72 \mathrm{~mm}$.

Dem kleineren Krümmungsradius vou $12 \cdot 5$ bis $14 \mathrm{~mm}$ Länge entspricht ine vordere Hauptbremweite der Hornhaut ron $3 \times 12.5$ bez. $3 \times 14=42 \mathrm{~mm}$.

Dem grösseren Krümmungsradius von $28 \mathrm{~mm}$ Länge entspricht eine wrdere Hauptbrennweite von $3 \times 28=84 \mathrm{~mm}$.

Sormit scheint die factische Wirkung der Hechthornhaut in Luft eher (in $: m$ mittleren Krümmungsradius zu entsprechen, als den beiden Extremen, obs:chon dieselbe allerdings dem längeren näher stelıt, als dem kürzeren.

Die ermittelte Kurzsichtigkeit des in Wasser getauchten Hechtauges berieht sich aber auf die rordere Netzhautebene, die Nerrenfaserschicht. Dises ist nicht die lichtpercipirende, musivische. Nehmen wir einen Tiefen-

"Ich brauche bei Fmmetropie des Untersuehten stets $-4 \cdot 5 D=-\frac{1}{9}-"$. Ich brauchte fir den Hecht -6 bis $7 \mathrm{~J}=-\frac{1}{5 \frac{1}{2}}$ bis $-\frac{1}{6 \frac{1}{2}}$ ". Das schwächere Conearglas ist für den Grad der Wyopie entscheidend, sowohl bei der subjectiven, wie bei der objectiven Refractionsmessung. 
abstand $\delta$ ron $0 \cdot 10 \mathrm{~mm}$ zwischen beiden und eine Brennweite der kugeligen Krystallinse ron etwa $8 \mathrm{~mm}$ an, so wäre in der That eine etwas stärkere Myopie rorhanden

$$
\mathscr{T}_{2}=\frac{F_{1} F_{1}}{\mathscr{T}_{1}}=\frac{8 \times 8}{0 \cdot 10}=640 \mathrm{~mm} .
$$

Es könnte die Myopie noch um etwa 1 bis 1.5 Dioptrien stärker sein, der Fermpunkt statt in $24^{\prime \prime}$ vielleicht in 16 Zoll liegen. Die Differenz zwischen scheinbarer und wirklicher Myopie des in Wasser getauchten Fischauges ist nicht bedentend. Dem entsprechend sieht der Beobachter ohne Aenderung seiner Accommodation gleichzeitig die Sehnervenfaserschicht ganz scharf im aufrechten Bilde, und auch die Andeutung der Aderhautstructur in genügender Schärfe. Die Constante $\mathrm{F}_{1} \times \mathrm{F}_{2}$ ist für das Hechtange (64) zwar kileiner als beim Menschen (300), aber doch erheblich grösser als beim Fruschauge (12).

Bei einem Hecht von $0.5 \mathrm{Kgrm}$. Gewieht, $30 \mathrm{~cm}$ Körperlänge, war die Hornhautbreite . . . . . . . . . . . . . . $13 \mathrm{~mm}$ die Pupillenbreite . . . . . . . . . . . . . . . . . . . . . . 6 " $r \mid$ a) am nasalen Rande des Pupillarbereiches . . . . . . . . . . . 7 ",

$r\{$ b) am temporalen Rande des Pupillarbereiehes . . . . . . . . . . 14,"

c) inmitten des Pupillarbereiches . . . . . . . . . . . . . . 12 ",

Das Reflexbild der Scheibe bei a regelmässig aus concentrischen Kreisen bestehend, bei $b$ und $c$ querelliptisch.

Myopie in Luft excessiv, Fernpunktsabstand . 2 Zoll "unter Wasser minimal " . . 24 Zoll

Bei einem grossen Hecht zeigt das rechte Auge eine Breite der Horn-

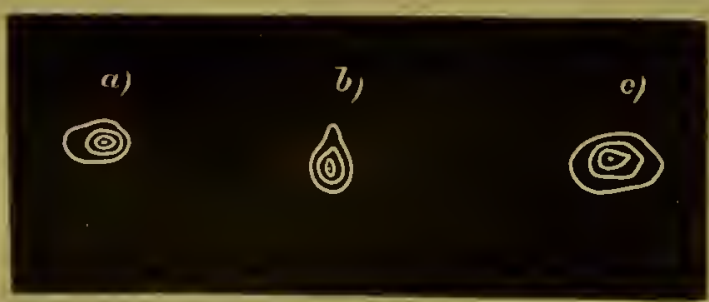

Fig. 3 a.

Hornhautreflexbilder des Kcratoskops beim Heeht.

a) von der Mitte des Pupillarbereiches.

b) vom nasalen Randc desselben.

c) voin temporalcn Rando desselben. (restaltung (Fig. $3 a$ ); an nasalen Rande des Pupillarbereiches $6^{\mathrm{mm}}$ hoch

1 Directc Messung von Dickendurchschnitten der Hechtretina ergab mir $\delta=0.2 \mathrm{~mm}$. Da ich aber auch hintcr der Sehncrvenfaserschicht gleichzeitig ein anderes Liviensystem mit genigender Schüre wahrnalım, so war der Apparat meines eorrigirten Auges doelı nicht mathematisch scharf auf die Selnervenfaserschicht eingestellt und die Annahme $\delta=0 \cdot 1 \mathrm{~mm}$ wohl zulïssig. 
und $t^{\text {nns }}$ breit (Fig. 36); an temporalen Rande des letzteren $8 \mathrm{~mm}$ breit und $5 \mathrm{~mm}$ hoch (Fig. 3 c); oberhalb des Pupillarbereiches $10 \mathrm{~mm}$ breit, $5 \mathrm{~mm}$ hoch und sehr unregeluässig. Der Krümmungsradius der Hornhaut ist etwil das Doppelte der Reflexbildlänge bez. Breite. Wir haben also die Wahl zwischen rerschiedenen Hornhautradien und können auch rom blossen duge leicht erkenuen, dass der im ganzen flachen Hornhaut des Hechtes ein sanft ansteigender Epicyclus ron stärkerer Krümmung im nasalen Theil des Pupillargebietes aufgesetzt erscheint.

Jedenfalls ist für ein Fischange, das dem menschlichen an Grösse nahekommt, der physiologisch wichtigste centrale Theil der Hornhaut mit einem horizontalen Krrümmungsradius ron $12 \mathrm{~mm}$ bez. $16 \mathrm{~mm}$, also mit einer Brechkraft ron $1 / 36$ bez. $1 / 18$ in Millimeterwerth ausgestattet.

Bei dieser Gelegenheit sei noch einer optischen Eigenthümlichkeit des Hechtauges gedacht, welche ron der Form und Lage der Krystallinse abhängt. Schon Sömmering d. J. und J. Müller haben darauf hingewiesen, dass bei Fischen die Conrexität der Linse über das Pupillargebiet in die Vorderkammer hineinrage. Es schien dies derzeit gewiss um so auffälliger, als man damals noch geneigt war, die menschliche Krystallinse hinter die Iris nach hinten zurücktreten zu lassen; erst die Anatomie am Lebenden, die wir den neuereu ron Helmholtz eingeführten Untersuchungsmethoden rerdanken, hat uns belehrt, dass der vordere Linsenpol in der Pupillarebene rles menschlichen Auges belegen ist.

Wer mit Hilfe des Augenspiegels das Hechtauge durchleuchtet, sieht den grossen mittleren Bereich der querovalen Pupille hell röthlich leuchten; dann folgt eine zartgrauliche Randzone der Krystallinse und nasenwie schläfenwärts der eigentliche Rand der Krystalllinse, welcher eine metallisch schimmernde Linie darstellt; der Zonularraum, der nasenwärts in Pupillarbereich frei liegt, erscheint ganz dunkel. Die Erklärung dieses Phänomens ist einfach. Oben nnd unten ist der Linsenrand wegen der inı rerticalen Durchmesser geriigeren Oeffnung der Pupille durch die undurchsichtige Iris rerdeckt. Rechts und links bleibt er frei. Aber wegen der vorgerückten Lage und Convexität der Krystallinse erscheint ihr lateraler Pand bei der Durchleuchtung wie der Rand der menschlichen Linse bei a uffallendem Licht, d. h. bei der seitlichen oder focalen Beleuchtung. In die aphakische Zone des Pupillargebietes kanu aber, wenn wir den Netzhautmittelpunkt als erleuchtet anselıen, überhanpt kein Theil des zurückkehrenden Lirchtes hineingelangen, da die 'Tangente zur Linsenkugel nach der Hinterfläche der Iris rielt. Blickt und leuchtet man schräg (ndsenwärts) in die Pup,ille hinein, so erscheint der linsenlaltige Theil der Intztren hellrïthlich, der Zonnlarraum bleibt dunkel; uur zeitweise gewinnt man einen schwar.h'n Lichts*himmcr aus demselben bei Drelıung des Spierrels 
Bei einer grossen Plötze war (am rechten Ange) die Horuhautbreite $=12 \mathrm{~mm}$, dic Breite der rundlichen Pupille $=6 \mathrm{~mm}$. Wnrde der lebende Fisch in Luft gehalten, so entsprach die Myopic des Auges nach der Augunspiegeluntersuchung einer lermpunktsdistanz ron etwa 2 Zoll. Das Reflexbild, welches der mittlere Theil der Hornliaut ron nnserer Scheibe lieferte, war etwa $4 \mathrm{~mm}$ breit, $3 \mathrm{~mm}$ hoch; im Ganzen etwas regelmässiger, als beim Hecht und nach dem Gesagten ron dem Reflexbild einer normalen menschlichen Hornhaut micht so erheblich an Grösse abwcichend. Der mittlere Theil der Hornhaut dieses Fisches hatte also Krünmungsradien, welche zwischen 6 nnd $8 \mathrm{~mm}$ schwanken.

Der obere Theil der Hornhaut entwarf von der Kreisscheibe ein Spiegelbild in Form einer rerticalen, der temporale Theil in Form einer horizontalen Ellipse, deren Längsaxe etwa $\delta^{\mathrm{mm}}$ lang war. Somit kommen in

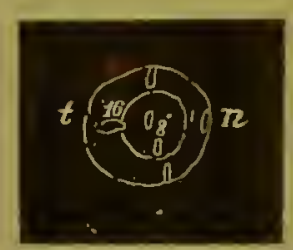

Fig, 4.

Reflexbilder der Hornhaut einer Plötze von dersclben Lichtflamme.

Zahlen = Krümmungsiadien . $n$ nasale Seite.

$t$ temporale Seite. den mehr excentrischen Partien dieserHornhant Krüwmungsradien ron $16 \mathrm{~mm}$ ror.

Eine klare Anschauung von den Krümmungsverhältnissen dieser Horuhaut giebt die schematische Fig. 4, welche die Reflexbilder einer Gasflamme an den rerschiedenen Stellen der Hornhaut ihrer Grösse nach darstellt. Mau erkennt, dass die im Ganzen flache Hornhant im inneren oberen Theil des präptupillaren Bereiches einen stärker gekrümnnten Epicyclns mit einem stark gekrüunmten Nabelpunkit entiält.

Aehnliche Bilder erhält mau am Menschen nur unter pathologischen Verhältnissen, im Fall des Keratoconus.

Bei einer kleinen Plötze war die Breite der Hornhant 10, der Pupille $5 \mathrm{~mm}$, des rom präpupillaren Theil der Hornhaut gespiegelton und ziemlich stark verzerrten Keratoskop-Reflexbildes auch $5 \mathrm{~mm}$, die Höhe derselben geringer $(r=10 \mathrm{~mm})$. Der Linsemrand nicht sichtbar, rohl aber unschriebene trübe Punkte in der Linse, was recht hänfig, ja meisteus bei Fischen beobachtet wird. Die objectir gemessene Mropic des lebenden Auges in Luft entspricht eincr Fempunktsdistanz von $1 \frac{1}{2}$ Zoll.

Bei ciner kleinsten Plötze war die Breite dor Horuhaut 7, der Pupille $4 \mathrm{~mm}$, die Breite des in der Mitte der Hornhaut gespiegelten KeratoskopReflexbildes $3 \mathrm{~mm}$; die Höhe desselben $2(r=6 \mathrm{~mm})$. Der Fermpunktsabstand des lebenden Auges in Luft etwa 1". Gerade hier war die Fiustellung auf das directe ungekehrte Augengrundbild eine genügend scharfe, da jedes Blutgefäss eine feine rothe Linie darstellte. 
Bei einem Aal ron $60^{\mathrm{cm}}$ Lünge war links die Hornhautbreite $5 \mathrm{~mm}$, Pupillarbreite $21, \mathrm{~mm}$. Trümmungsradius der Hornhant $5^{1} / 2$ bis $7 \mathrm{~mm}$ inmitten des Pupillargebiets. Das Reflexbild war stark rerzerrt, - natürlich auch das Augengrundbild in Luft; starke Myopie dabei zu constatiren. Atropin und Eserin wirken nicht merkhar auf die Pupille.

Ziehen wir das Facit aus diesen Beobachtungen am lebenden Fischauge. Die Fischcornea, die im Wasser nicht gebraucht wird, ist ebensowenig ausgearbeitet; wie die dem Beschauer abgewendete Seite der Giebelstatuen altgriechischer Kïunstler. Im Ganzen ist die Hornhaut flach im Terhältuiss zur Sehaxenlänge. Bei einem Hechtange, dessen Hornhautbreite gleich der des Menschen, dessen Sehaxenlänge hall so gross wie die des Menschen, kommen im Pupillargebiet Krümmungsradien vor, die über doppelt so gross sind wie die des Menschen; jedoch ist der nasale Theil des präpupillaren Gebietes merklich stïrlker gelirümmt als der temporale.

Nichtsdestoweniger wäre es ungereimt, die optische Wirkung der Hornhaut. wemn das Fischauge in Luft taucht, gleich Null zu setzen. Sie ist bei derartigen Hechtaugen immer noch fast so gross wie die der Krystalllinse des menschlichen für seinen Fernpunlit accommodirten Auges. Ehenso wie der Terlust der Krystallinse ein normales Menschenange stark übersichtig macht $\left(\mathrm{H}^{1}{ }^{\prime \prime}{ }^{\prime \prime}\right)$, wird das Fischauge, sowrie es in Luft emportaucht, durch Inkrafttreten der Hornhautbrechung stark kurzsichtig. Der Fernpunkt liegt $1-3$ " vor dem Fischauge, je nach der Grösse des letzteren. Dies lehrt die objective Refractionsmessung mit Hilfe des Augenspiegels.

Der Betrag dieser Kurzsichtigkeit wird nahezu gedeckt durch die Brechkraft der Hornhaut, wie sie sich aus dem Krümmungsradius der Hornhaut annäherud berechnen lässt. Hiernach ist zu rermuthen, dass die Refraction des in Wasser getauchten Fischauges nur wenig ron dem normalsichtigen, emmetropischen Zustand abweichen werde.

Die directe Beobachtung mit dem Augenspiegel bestätigt die Vermuthung in unwiderleglicher Weise. Das in Wasser getauchte Fischauge ist aher nicht rollkommen normalsichtig, sondern leicht kurzsichtig. Der Fernfrunkt des Hechtauges liegt etwa in 24 Zoll, vielleicht etwas näher, rinlleicht anch nicht. Die optischen Bilder des in Wasser getauchten Auges rom Hecht sind recht gute. Wir verstehen in optischer Hinsicht liẹ kraftvolle Action dieses kühnen Räubers; auch ein Mensch mit einem ahulirh geringen Grade von Iyopie sieht recht gut für alle gewöhnlichen Verrichtungen, selbst ohne Concarglas.

Hr. Plateau schenkt dem Hecht in Wasser eine Fernpunktsdistanz

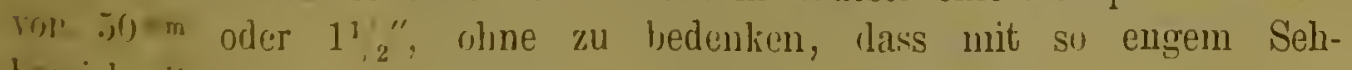
bertich die Hechte wohl lange im Kímpf umis Dasinin zu Grunde gegangril wären. 
Ich bemerke übrigens noch, dass rom teleologischen Standpunkte aus eine mässige Kurzsichtigkeit der Fische nicht unzweckmässig scheint. Auch das klarste Wasser ist auf grössere Strecken undurchsichtig.

\section{$R w$}

\section{Fig. 4 a.}

Schema des Strahlenganges im Hechtauge (nach dem Verfasser).

$R l$ Feropuulit in Luft.

$R w$ Fernpunkt in Wasser.

$\overline{C R l}$ und $\overline{C R n}$ sind auf $1 / 6$ reducirt; das Auge hat seine richtigen Dimensionen. $N$ ist die Netzhaut, $S$ Sclera, $C$ Cornea.

Das von einem Netzhautpunkt ausgehende Strahlenbündel ist im Karnmerwasser nahezu parallel.

Iatthiessen's Fischaugen in Wasser sind emmetropisch; doch ist eine Constante, der totale Brechungsindex der Linse berechnet und nicht beobachtet; jedenfalls mögen seine Zahlen hier eine Stelle finden, um Pla te au's Anschauungen zu entkräften. Matthiessen (Dioptrik. 1877. S. 217) fand beim Seebarsch

$r$ der Hornhaut . . . . . . . . . . . . . . $6.6 \mathrm{~mm}$

$r$ der beiden Linsenflächen . . . . . . . . 2.5"

Axe und Durchmesser der Linse . . . . . . . 5.0 "

Ort der vorderen Linsenfläche . . . . . . . . 0.5 "

" des Linsencentrums . . . . . . . . . . . 3.0"

" der hinteren Linsenfläche . . . . . . . . . 5.5 "

"der Retina . . . . . . . . . . . . . . $8.5 "$

Brechungsindex der Hornhant . . . . . . . 1.38 "

$" \quad$ des Glaskörpers . . . . . . . 1.335",
$" \quad$ " $\quad$ mittleren Linsenschicht . . . . . $1.49 "$
$" \quad$ des festen Kerns . . . . . . . $1.5 "$

Wr berechnet den totalen Brechungsindex der Linse $n=1.7 "$ und die Brennweite $\mathrm{F}_{1}$ der Linse im Glaskörper . . $5.5 "$

Es ist nicht wahrscheiulich, dass den Fischen eine accommodative Linsenverdickung gegeben ist, wie dem Menschen, der übrigens im ganzen 
Thierreich relatir die flachste Linsenform besitzt. Die Fischlinse ist kugelig, l. b. ungünstig für eine Verlängerung in Richtung der Sehaxe; auch zu lest, un rasch ilme form zu ändern. ${ }^{1}$

Was die a uatomischen Yerhältnisse der Fischaugen anbetrifft, so lehrt schon die makroskopische Präparation des Hechtauges Folgendes: 1) Beim dequatorialschnitt sieht man, dass die kugelige Krystallinse einen rerhältnissmässig lileinen Umfang besitzt, dessen Kreis die Ellipse der Pupille am obereu und am unteren Rande ron innen her berührt, während zwischen dem nasalen und temporalen Pupillarrande einerseits und dem entsprechenden Linsenrande andererseits ein freier Raum (Zonularraum) übrig bleibt, der übrigens vollkommen durchsichtig ist. Der Glaskörper ist nur eine dünne fast membranöse Schicht. 2) Auf dem Horizontalschnitt stellt die Hornhaut gewissermaassen eineu Concavmeniscus dar, indem ein solides Ligamentum pectinatum iridis sich ihrer Hinterfläche anschmiegt. Dies ist schon im Anfange unseres Jahrhunderts von Rosenthal (Archiv f. Physiol. ron Reil und Antenrieth. Bd. X, S. 398) angemerkt und von Dr. W. Sömmering (De ocul. hom. animaliumque. Gott. 1818) sorgfältig abgebildet worden. Im Ganzen wird der Meniscus wenig ausmachen, doch kann dadurch die Myopie ein wenig geringer sein, als sie bei parallelen Hornhautflächen betragen würde. ${ }^{2}$

1 Aber unmöglich ist es nicht, dass Fische doch eine Art von Accommodation besitzen, nämlich durch Lageveränderung der Krystallinse.

Dicke der Linse zur Breite derselben

$$
\begin{aligned}
& \text { beim Menschen (puella XX annor.) }=1 \cdot 6^{\prime \prime \prime}: 3 \cdot 6^{\prime \prime \prime}=1: 2 \cdot 5 \\
& \text { bei Simia Inuus . . . . . }=2 \cdot 0: 3 \cdot 4=1: 1 \cdot 7 \\
& \text { "Canis Isupus . . . . . }=3 \cdot 9: 5 \cdot 4=1: 1 \cdot 4 \\
& \text {,Phoka groenl. . . . . }=4.6: 5 \cdot 0=1: 1 \cdot 1 \\
& \text { "Falco chrysaetos.... . }=3 \cdot 8: 5 \cdot 7=1: 1 \cdot 5 \\
& \text { "Anas cygnus . . . . . }=3 \cdot 0: 3 \cdot 8=1: 1 \cdot 26 \\
& \text { "Rana teinporia. . . . . }=1 \cdot 6: 2 \cdot 0=1: 1 \cdot 25 \\
& \text { " Lacerta monitor. . . . . = } 1 \cdot 7: 2 \cdot 4=1: 1 \cdot 4 \\
& \text { "Coluber Aescul. . . . . . }=1 \cdot 4: 1 \cdot 4=1: 1 \\
& \text { "Esox lucius . . . . . }=3 \cdot 4: 3 \cdot 4=1: 1 \text { (Socmmering.) }
\end{aligned}
$$

Auch bei den Säugethieren und Vögeln haben die Wasserbewolner cine nahezu k: gelige Linse $(1: 1)$.

IIatthiessen (Dioptrik. Icipzig 1877. S. 217) fand den Brechungsindex der Hombant des Seebarsches and des Dorsches gleich 1.38. Den Krümmuugsradius der vorleren Hornhautfläche des Hechtes nach Sömmering's Abbildung zu 14, den der liogs zr 4, den der hintcron Hornhautfliche mit ciniger Annälicrung zu $9 \mathrm{~mm}$ gesetzt, die Licke der Hornhant zu $1 \mathrm{~mm}$ : so hätten wir dic Brennweite des auf die benetzende Wasserschicht an der Hornhautoberflächc folgenden Concnvmeniscus

$$
\psi=\frac{n_{1}}{\left(n_{2}-n_{2} r_{1}\right) n_{2}} \frac{r_{2}}{\left.\left(r_{2}-r_{1}\right)+\left(n_{2}-n_{1}\right) d\right]}=-7339 \mathrm{~mm}
$$


Hinsichtlich der Maasse eines grossen Hechtauges sei folgender Fall hervorgehoben :

Iänge des Auges $15 \mathrm{~mm}$, Breite $20 \mathrm{~mm}$

" der Linse $7, \quad$ " 7 "

Abstand der Netzhaut von der Torderfäche der Hornhaut (Sehaxenlänge) $\cdot 12 \mathrm{~mm}$.

Bein Fischauge besteht wegen der Choroidaldrüse ihres Analogous immer ein rerhältnissmässig beträchtlicher Unterschied zwischen Bulbus und Sehaxenlänge (15:12 mm, - beim Menschen 24:23 mm!)

Für ein Plötzenauge fand ich:

Länge des Auges $7 \mathrm{~mm}$, Breite $10 \mathrm{~mm}$

$$
" \text { der Linse } 3 \text { " } " 3 \cdot 5,
$$

Glaskïrperraum sehr eng.

Sömmering's Zeichnung ergiebt für ein sehr grosses Hechtange eine Sehaxenlänge von . . . . . . . . . . . . $15 \mathrm{~mm}$ Augenaxenlänge . . . . . . . . . . . . . 20 " Horuhautbreite . . . . . . . . . . . 20 " Hornhautdicke . . . . . . . . . . . . . 1 "im Centrum Vorderkammertiefe . . . . . . . . . . . $<1$ " Linsenlänge . . . . . . . . . . . . . $71 / 2:$ Linsenbreite . . . . . . . . . . . . . . $71 / 2 \%$ Abstand zwischen hinterem Linsenpol und Tetzhaut. 5 " Netzhautdicke . . . . . . . . . . . $<1 / 2$ "

Sömmering's Beschreibung des Hechtauges lautet im Auszug:

Bulbus magnus, horizontaliter paullum oblongus. Corneae facies anterior cum posteriore minime concentrica. Choroildea admodum criassa. Nervus opticus crassus; nervi medulla externe sclerotices et choroïdeae crassitiem flexuose penetraus, in caudam quasi ut in aribus complanata, originem praebet retinae forti. Ex hac nerri iusertione processus quoque excurrit falciformis niger, versus marginem ureae inferiorem tendens, ${ }^{1}$ ubi in campanulam Halleri prominentem pigmento

Aber wir kennen $r_{2}$ nicht genau genug und $r_{1}$ ist $z u$ hoch gegrifien. Auch die menschliche Hornhaut ist eigentlich ein Concavmeniseus vou etwa $8700 \mathrm{~mm}$ Brennweito. (Holmholtz, Physiologische Optik. S. 71.)

${ }^{1}$ Vgl. unten die opthalmoskopische Beschreibung. 
nigro obductam, intus vero albam terminatur. Isenti campanula haec subtus affixa. in superiore rero parte ligamento albido latiusculo satis firmo cum ritreo corpore comnectitur Lens globosa, hemisphaerio suo toto super iridem eminens, corneae quamris proxima, non tamen ei presse accumbit neque pupillie aream implet, ita ut radii lucis multi inter lentem et pupillae limbuu ad retinam perreniant. Humor aqueus paucissimus, vitreus parrus.

Vergleichen wir das Auge des lebenden Frosches mit dem des Fisches (Hechtes).

Der Augapfel des Frosches ist nahezu liugelrund, der des Fisches queroral. Die Hornhaut des Frosches ist durchaus regelmässig und genügend gewülbt, der Kirümmungsradius nahezu gleich der halben Sehaxe; die Hornhaut des Fisches ist durchaus unregelmässig und dabei flach gewölbt, der Krrümmungsradius gleich der doppelten Sehaxe und darüber. Die Einstellung des Froschauges in Luft ist scheinbar hypermetropisch, die des Fischauges in Luft stark myopisch; das ron den brechenden Medien selbst entrorfene Bild der Netzhautrorderfäche liegt einige Zoll hinter dem Froschauge in Luft und ist aufrecht, wenige Zoll vor dem Fischauge in Luft und ist umgekehrt: ein grösserer Unterschied der Einstellung kann kaum gedacht werden.

Bezüglich des Froschauges ist die bemerkenswerthe Thatsache zu constatiren, dass ihm jede Spur jener accommodativen Aenderung der Linsenwōlbung fehlt, die wir beim Menschen so bequem objectiv nachweisen können.

Trāufeln wir Atropinlösung in das gesunde Menschenauge, so wird dasselbe durch Abflachung der Krystalllinse für seinen fernsten Punkt eingestellt; träufeln wir Eserinlösung ein, so wird das menschliche Auge durch stärkere Wölbung der Krystalllinse auf seinen nächsten Punkt fest eingestellt.

Das Froschauge hat, wie die objective Untersuchung mit dem Augen- piegel lehrt, dieselbe Refraction vor wie nach der Atropin-, bez. EserinEinträufelung. Nach den Anatomen fehlt dem Froschauge der Ciliarmuskel. [-. (r),rigens ist seine Krystalllinse ziemlich hart, fast kugelig und füllt den Grösten Theil des Bulbusraumes aus; folglich ist jede stärkere acconmodatice Schwankung der Linsenform fast auszuschliessen.

Stellt ein Beobachter sein Auge mit Hilfe des Ophthalmoskops genau anf rlie Sehnervenfaserausstrahlung am unteren Rande der Papille ein, was bej der Sirhärfe des Bildes sehr gut angeht, so findet er regelmässig eine whinhare Hypermetropie des in Luft getauchten Froschauges, welche je nach der Gröss; des lotateren zwischen $\frac{1}{8}$ " und $\frac{1}{5} "(=5-7 \cdot 5$ Diopt. $)$ whwankt: l. h. das schrinhar von einem Punkte der Netzhautvorderfläche all-grahrnde Strahlenbündel verlässt das Froschauge divergent; in der Weise,

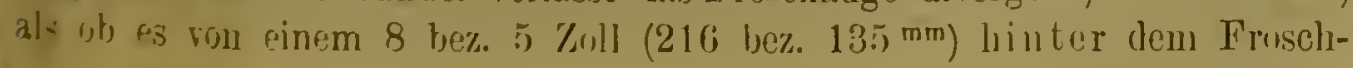


auge gclegenen Punlit ausginge. Bei den grösseren (ungarischen) Fröschen ist jenc scheinbare Hypermetropie etwas geringer $\left(\frac{1{ }^{\prime \prime}}{8}\right)$ als bei den kleineren gewöhnlichen $\left(\frac{1}{5}{ }^{\prime \prime}\right)$.

Beim Menschenauge liefert die Einstellung auf den (temporalen) Sehnervenrand auch die wirkliche Rcfraction: wenigstens ist der Fehler unbedeutend, den der Tiefcuabstand zwischen der beim Augenspiegeln lichtreflectirenden und der von uns nicht wahrnehmbaren, beim Sehact lichtpercipirenden Fläche der Stäbchen- und Zapfenschicht einführt: ein positiver Abstand von $0.3 \mathrm{~mm}$ würde eine scheinbare Hypermetropie von

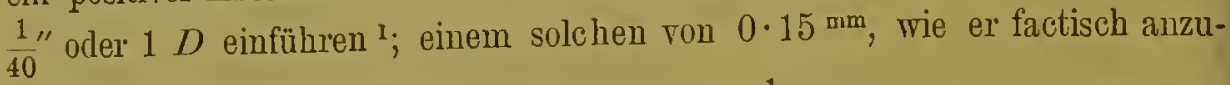
nehmen, entspräche eine Hypermetropie ron $\frac{1}{80}$ ", d. h. das schwächste in unserem Brillenkasten vorfindliche Glas, dessen optische Wirkung fast unmerklich ist. ${ }^{2}$

Anders liegen die Verhältnissc für den Frosch. Es ist eine höchst bemerkenswerthe Thatsache, dass, während die Sehaxe auf $1 / 3$ bis $1 / 4$ der menschlichen (nämlich auf $6-8 \mathrm{~mm}$ ); die Constante $\mathrm{F}_{1} \mathrm{~F}_{2}$ auf $\frac{1}{25}$ des Terthes beinı Menschen (עämlich auf $3 \times 4=12)^{3}$ reducirt ist, die Netzhautdicke von derselben Grössenordnung (etwa $0 \cdot 3 \mathrm{~mm}$ ) geblieben.

Setzen wir beim Froscli $\varphi_{2}=-12 \mu=-0.012 \mathrm{~mm}$; so wird $\varphi_{1}=\frac{-12}{0.012}=\frac{-12 \times 1000}{12}=-1000 \mathrm{~mm}$. Die scheinbare $H$ wird $\frac{1}{40}$ $(=1 \mathrm{D})$, wozu beim Menschen der Tiefenabstand 25 Mal so gross $(=300 \mu)$ sein müsste.

Dass sehr geringe Tiefenabstände in der Froschnetzhaut schon beträchtliche Refractionsunterschiede bedingen, ist schr leicht nachzuweisen; hat der (nicht accommodirende) Beobachter durch ein passendes Glas hinter dem Augcnspiegel sich auf die Sehnerrenfaserschicht der Froschnetzhaut

' $F_{1} F_{2}=\tau_{1} r_{2}$ giebt für das reducirte Menschenauge $15 \times 20=\tau_{1} q_{2} ;$ setzen wir $q_{1}=-1000 \mathrm{~mm}$, so wird $m_{2}=\frac{300}{-1000}=-0.3 \mathrm{~mm}$ : w. z. b. w.

${ }^{2}$ Weit grösser ist mituntcr, bci unregelmässiger Form des menschlichen Augapfels, der Unterschied des Radius vector, der von Knotenpunkt zum Sehncrvenrand eincrscits und rom Knotenpunlit zur Fovea andererscits gezogen werden kann.

${ }^{3} F_{1}=3 \mathrm{~mm}$ wach Kühne's Optographic und meincr Ophthahnomikrometric. Die Länge der Froschpapille hatte ich zu 0.5 angenoumen; die genauere Mikrometric ergicbt mir $0.455 \mathrm{~mm}$. - In der Note S. 83 dieses Jahrgangs ist ein Rechenfehler uncorrigirt gcblieben. 
scharf eingestellt, so muss er das gefundene Correctionsglas noch mindestens um $+\frac{1}{10}$ bis $\frac{1}{s}$ " verstïrlien, damit der Blutstrom der so dümen Tena hyaloides in rollster Deutlichkeit erscheine.

Um die Hrpermetropie von $\frac{1}{5}$ bis $\frac{1}{8}$ in Zollmaass ( 8 bis 5 Dioptrien) beim Frosch fūr eine scheinbare zu erklären, bedürfen wir nur der Annahme, dass die wirklich lichtauffangende Fläche $5 \times 0.012$ bis $8 \times 0.012$ $=0.06$ bis $0.096 \mathrm{~mm}$ hinter der Schnervenfaserschicht liegt. Dieser Abstand ist aber thatsächlich noch grösser. Nach meiner Messung liegt die musivische Schicht etwa $0.2 \mathrm{~mm}$ hinter der Sehnervenfaserschicht in der Nähe der Papille. Nichts himdert uns, das Froschauge in Luft für myopisch zu halten.

$$
\begin{gathered}
\varphi_{1}=\frac{F_{1} \times F_{2}}{p_{2}}=\frac{3 \times 4}{0.2}=60 \mathrm{~mm} \\
\frac{1}{60} \mathrm{~mm}=+16 \text { Diopt. }
\end{gathered}
$$

Wird dieser Betrag der scheinbaren Hypermetropie von 8 Dioptrien hinzugefügt, so bleibt eine wirkliche Myopie von 8 Dioptrien oder $1 / 5$ in Zollmaass. Dass der Frosch in Luft gut sieht und nicht bloss auf ganz kurze Distanzen, lehrt die Beobachtung, - wie geschickt er die Fliegen im Sprunge erhascht.

5 Zoll sind $135^{\mathrm{mm}}$. Ich will keineswegs behaupten, dass die Fernpunktsdistanz des Frosches in Luft nicht grösser sein könne. Aber für noch hleiner mōchte ich sie nicht halten, und den Werth des Hrm. Plateau $(35 \mathrm{~mm})$ halte ich für ganz unwahrscheinlich. Kühne erhielt bei einer Dbjectdistanz ron $250 \mathrm{~mm}$ scharfe Optogramme auf der Netzhaut des eben getridteten Frosches; auch dieses Resultat stimmit doch noch eher mit meinen Anschauungen als mit Plateau's Zahlen überein.

Wollten wir uns einen Augenblick auf den teleologischen Standpunkt stellen, so könnten wir sagen, dass für den Frosch, - schon um seine ejgenen Glieder einigermaassen deutlich zu sehen, eine gewisse Kurzsichtighait zweckmässiger sei, als die Emmetropie, die den Menschen ziert. ${ }^{1}$

Auch ohne jede accommodative Linsenverdickung wird die Accommodatirnshinie beim Frosch länger sein, als bei einem auf dieselbe Entfernung eings-trilten Menschen aus einem doppelten Grunde, einem dioptrischen und anem histologischen: die kurze Brennweite des dioptrischen Systems beim Frrech muss die Bilder der in ziemlich verschiedenen Entfernungen, vor und hinter seiner Fernpunktsebene, belegenen Gegenstände in eine kurze streck: dor Sehaxe zusammendrängen; die relative Breite der musivischen

Ios homini sublime dedit crelumque tueri jussit. O)id. 
Elemente ${ }^{1}$ (bis $7 \mu$ ) muss absolut grössere Zerstreuungskreise eines leuchtenden Punktes zulassen, ehe die Wahruehmungsfähigkeit aufhört. ${ }^{2}$

Die Hornhaut des Frosches hat im Pupillargebiet einen Krümmungsradius von etwa 4 bis $5 \mathrm{~mm}$ und ist ausserordentlich regelmässig gewölbt, genau so wie beim Menschen. Ton einer Abplattung in der Hornhantmitte ist keine Spur am lebenden Frosch mit Hilfe der optischen JLethuden zu entdecken. Die rordere Hauptbrennweite des brechenden Hormhautsystems ist $F_{1 c}=3 \times 4$ bis $3 \times 5=12$ bis $15 \mathrm{~mm}$. Der Ausfall eines solchen Systems muss recht merkbar seiu.

Wäre das Froschange in Luft factisch wahezu normalsichtig, so müsste dasselbe, in Wasser getaucht, stark übersichtig werden und würde, um die Normalsichtigkeit beizubehalten, eine positive Taucherlinse brauclien, der in Wasser eine Brennweite von etwa $1 / 2$ Zoll zukommt, wie der Mensch unter Wasser eine solche von etwa $1^{1 /} / 4$ bis $1 \frac{1}{2}$ " braucht.

Ist der Frosch in Luft kurzsichtig (mit Myopie $\frac{1}{8}$ " oder $\frac{1}{5} "$, Ferupunktsdistanz $216 \mathrm{~mm}$ oder $135^{\mathrm{mm}}$ ), so wird er in Wasser nicht bloss weniger kurzsichtig, sondern immer noch sehr stark übersichtig sein, da $\frac{1}{216}$ und selbst $\frac{1}{135}$ gegen $\frac{1}{12}$ nur wenig in Betracht kommt.

${ }^{1}$ Beim Menschen sind die Zap fen $32=36 \mu$ lang, $4 \cdot 5-6 \mu$ breit, (in der Fovea $60-100 \mu$ lang, $3 \mu$ breit, an der Spitze sogar nur $1 \mu$ )

Beim Fisch sind die Zapfen länger als beim Menschen.

Beim Frosch sind die Zapfen $20 \mu$ lang, $5 \mu$ breit.

Beim Menschen sind die Stäbchen $60 \mu$ lang, $2 \mu$ breit.

Beim Fisch sind die Stäbchen $74 \mu$ (und mehr) lang. $2 \cdot 5 \mu$ breit.

Beim Frosch sind die Stäbchen $6-7 \mu$ breit.

2 Es sei für den Menschen $F_{1} \times F_{2}=300$, für den Hecht in Wasser $=8 \times 8=64$. für den Frosch in Luft $=3 \times 4=12 ; r_{1}$ sei die Object-, $r_{2}$ die Bilddistanz, von den Hauptbrennpunkten ab gerechnet. Die Pupillenbreite $p$ sei $4 \mathrm{~mm}$ für den Mensch, $7 \mathrm{~mm}$ für den Hecht, $4 \mathrm{~mm}$ für den Frosch. Dann ist die Breite der Zerstreuungskreise eines Lichtpunktes $\mathrm{Z}=p \frac{F_{1}}{F_{1}+p_{1}}$, wenn das Auge emmetropisch vorgestellt wird. (Vgl. Helmholtz, Physiologische Optik. S. 100.) Man erhält die folgende Tabelle.

\begin{tabular}{|c|c|c|c|c|c|c|c|c|}
\hline \multicolumn{3}{|c|}{ Mensch. } & \multicolumn{3}{|c|}{ Hecht. } & \multicolumn{3}{|c|}{ Frosch. } \\
\hline r 1 & $r_{2}$ & $\mathrm{Z}$ & $r_{1}$ & $r_{2}$ & $\mathrm{Z}$ & $r_{1}$ & $r_{2}$ & Z \\
\hline$\infty$ & 0 & $0 \mathrm{~mm}$ & $\infty$ & 0 & 0 & $\infty$ & 0 & 0 \\
\hline 65000 & $0 \cdot 05$ & $0 \cdot 0011$ & 65000 & $0 \cdot 0009$ & $0 \cdot 0008$ & 65000 & $0 \cdot 0001 \mathrm{~s}$ & 0.00018 \\
\hline 12000 & 0.025 & 0.0056 & 12000 & $0 \cdot 005$ & 0.0046 & 12000 & $0 \cdot 001$ & 0.0011 \\
\hline 3000 & $0 \cdot 100$ & $0 \cdot 0222$ & 3000 & $0 \cdot 021$ & 0.0119 & 3000 & 0.004 & 0.004 \\
\hline 750 & $0 \cdot 40$ & $0 \cdot 08$ & 750 & 0.08 & 0.074 & 750 & 0.016 & $0 \cdot 016$ \\
\hline 94 & $3 \cdot 2$ & 0.5 & 94 & $0 \cdot 7$ & 0.55 & 94 & 0.12 & $0 \cdot 12$ \\
\hline
\end{tabular}


Das beim Eintauchen des Froschauges in Wasser die Refraction des tuges sich stark verringert, lehrt der Versuch. Ich sah mit einem Convexglis ron $\tau^{1} / 2$ " Brennwreite $(=5 D)$ hinter dem Spiegel im aufrechten Vetzhautbilde den Gefässbaum der Vena hyaloildes; träufelte einige Wassertropfen auf die Cornea und legte (ohne jeden Druck) ein kleines Stückchen eines Deckgläschens auf: jetzt brauchte ich ein Correctionsglas von $+21 / 2$ $(=16 D)$ hinter dem Spiegel, um den Gefässbaum einigermaassen deutlich zu erkennen; doch fehlten mir noch stärkere Gläser zum Vergleich; das Bild war micht ganz scharf und stark verkleinert. ${ }^{1}$

Soriel ist sicher, dass in anatomischer Hinsicht durch die Wölbung der Cornea, in functioneller durch die scheinbare Hypermetropie das Froschauge sich dem Auge der Landthiere nähert, während allerdings die kugelige Wölbung seiner Krystalllinse wieder mehr dem Fischauge zu entsprechen scheint.

Wahrscheinlich ist, dass wir dem Froschauge in Luft eine einigermaassen kurzsichtige Refraction zuschreiben müssen; die Netzhautdicke $\left(0 \cdot 2^{\mathrm{mm}}\right)$ kommt schon gegenüber der kurzen Brennweite des Systems $\left(F_{1} \times F_{2}=12\right)$ erheblich in Betracht: so sicher man auch beim lebenden Frosch die Refraction für die Tetzhautvorderfläche bestimmen kann, die musivische Schicht, auf welche es hierbei functionell allein ankommt, kaun man nicht am lebenden Auge mit dem Spiegel einstellen.

Es erübrigt noch, einige der an Froschaugen angestellten Messungen in Kürze mitzutheilen. Bei einem gewöhnlichen Frosch, dessen Augengrundsbild das Eigenthümliche hatte, dass die Arteria hyaloïles nicht weit ron der Papilla (nasenwärts) rerlief, und deshalb zu der Anfertigung unserer schematischen Zeichnung benutzt wurde, war die Breite der Hornhaut $i^{\text {enro }}$, der Pupille $4 \frac{1}{2} \mathrm{~mm}$, des im Verhältniss zum Fischauge sehr regelmässigen Scheibenreflexbildes $2^{\mathrm{mm}}$, also der Krümmungsradius der Hornhaut etrra $4 \mathrm{~mm}$. Die scheinbare Hypermetropie war $\frac{1}{8}^{\prime \prime}=5 D$, wenn ich für das Sehnervenfaserbündel einstellte, welches rom unteren Rande der Papilla schläfenıärts ausstrahlt. Ich träufelte in das rechte Auge von einer $1 / 2 \%$ Atropin-, in das linke von einer $1 / 2 \%$ Eserinlösung. Nach 'inigen Stunden war beiderseits die Pupille ein wenig enger als zuvor, Wenfalls kein merkbarer Unterschied zwischen beiden Pupillen und beidersits die scheinbare Hypermetrupie ein wenig crhöht (auf $\frac{1}{5} "=8 D$ ), aber

1 Ja ich Hypothesen nicht erörtern will, lasse ieh ganz dahin gestellt, ob del. Frrseh überhaupt einen Accommorlationsapparat besitat, un diesen Defeet au Brechung ant $r$ Wasser auszagleiehen, ob er z. B. seinen aus der ()rbita frei hervorragenden Augapfel durch Jrack seitens der Jider zu verlängern im Stande ist. Es wäre wünschens. werth, den froseh bein Schwinmen genauer zu beobachten, als mir dies möglieh ist. 
zwischen beiden Augen kein merklicher Unterschied. (Die Einstellung auf die Vena hyaloides gab noch eine stärkere $H$, von 13 D.)

Ein zweiter Frosch zeigte:

Breite der Hornhaut

" " Reflexbilder von der Hornhaut 2 (also $r=4^{\mathrm{mm}}$ ); der Pupille 4 "

Die scheinbare Hypermetropie war $1 / 8^{\prime \prime}=5 \mathrm{D}$. In das rechte Auge wurde Atropin, in das linke Eserin geträufelt.

Nach einigen Stunden war die scheinbare Refraction dieselbe wie zuror und gleich für beide Augen. Die scheinbare Refractionsdifferenz für Einstellung auf die Vena hyaloïdes und auf die Sehnervenfasernschicht betrug $+6 D$.

Am folgenden Tage war die Atropinpupille $4^{\mathrm{mm}}$ breit die Eserinpupille $3 "$,

Die scheinbare Refraction wenig geändert $\left(\right.$ Hto $\left.\frac{1}{6^{1 / 2}} "=6 \mathrm{D}\right)$ und gleich für beide Augen.

Bei einem gewöhnlichen Frosch war beiderseits

$$
\begin{aligned}
& \text { die Breite der Hornhaut. . . . . } 7 \mathrm{~mm} \\
& " \text { " . Pupille. . . . . } 4 \text {. bei Gashicht; } \\
& \text { " . des Scheibenreflexbildes fast } 2 \text { " } \\
& \text { " " Fensterreflexbildss . . } 0.5 "
\end{aligned}
$$

Danach berechnet sich $r$ auf 3.5 (bis 4$)^{\mathrm{mm}}$. Die Spiegelung der Hornhaut im praepupillaren Theil war durchaus so regelmässig wie beim normalen Menschenauge. Die scheinbare Hypermetropie betrug $\frac{1}{5}$ " $(S D)$ für die Sehnervenfaserung, $\frac{1}{3}{ }^{\prime \prime}(13 D)$ für die Vena hyaloïdes; beiderseits gleich.

Rechts Atropin, links Eserin eingeträufelt, nach $1^{\text {h }}$ dieselbe Refraction. Einträufelung wiederholt.

Am folgenden Tage dieselbe Refraction. Dieser Versuch wurde bei einem Sommerfrosch wiederholt und genau dieselben Zahlen und Resultate gewonnen.

Bei einem grossem (ungarischen) Frosch war die Breite der Hornhaut . . . . . $91 / 2 \mathrm{~mm}$ ". " $\quad$ " Pupille . . . . . . . 6 " (bei Gaslicht) $"$ " des Scheibenreflexbildes . . $2^{1 / 2}$, im praepupillaren Theile der Hornhaut. $\left(r=5^{\mathrm{mm}}\right.$.)

Das Reflexbild der Fensterbreite betrug $3 / \mathrm{mm} . \quad(r=5 \cdot 2 \mathrm{~mm}$. 
Die scheinbare $H$ betrug $5 D=\frac{1}{8}$ (Zoll), weun man für die Sehnervellfiserausstrahlung an unteren Rande der Papille einstellte. Um den Doppelstrom in der Vena hyaloides am unteren Sehnervenrande scharf wahrzunehmen, war ein um 3 bis $4 \mathrm{D}\left(=\frac{1}{12}\right.$ bis $\left.\frac{1}{10}\right)$ stärkeres Convexglas erforderlich. Am linken Auge desselben Thieres erhielt ich dieselben Werthe: die Pupille war im freien Tagesliclit etwa $5.5 \mathrm{~mm}$ breit; ein Wechsel der Pupillenbreite bei abwechselnder Belichtung und Besschattung, wie beim gesunden AIenschenauge, honnte nicht constatirt werden. ${ }^{1}$

Auf das rechte Auge wurde Atropinlösung $(1 / 2 \%)$ reichlich getupft, auf das linke Eserinlösung $(1 \%$ ). Nach einer Stunde, wo bei einem gesunden Menschen die Pupille des rechten Auges etwa $8-9 \mathrm{~mm}$, die des linken etrra $1^{\mathrm{mm}}$ breit geworden, und das rechte auf seinen absoluten Fern-; das linke auf seinen Nahepunkt festgestellt sein würde, konnte ich an den Pupillen und in der scheinbaren Hypermetropie, also in der Refraction, einen Cnterschied nicht wahrnehmen.

Die Einträufelungen wurden wiederholt; am folgenden Morgen war die Pupillenbreite und die scheinbare $H$ wie vor der Einträufelung.

Die anatomische Untersuchung des Auges rom grossen (ungarischen) Frosch ergab am Horizontalschnitt:

Breite des Auges (des Aequators) $=10 \mathrm{~nm}$

Länge " " . .. $81 / 2 "$

Hornhaut gut gerrölbt.

Torderkammer eng.

Breite der Linse $6 \mathrm{~mm}$

Dicke :" " $\quad 5^{\mathrm{mm}}$, also mehr als beim Menschen.

Die Linse füllt fast den ganzen Bulbusraum; zwischen ihr und der Retima bleibt ein mit Glaskiörper gefüllter Spalt, der nicht viel breiter ist als die Vorderkammer.

Ich mass an einem Dickendurchschnitt rom gehärteten Froschange, welcher Papilla und angrenzende Retina enthält, in letzterer

die Dicke der musirisehen Schicht . . . $=74 \mu$

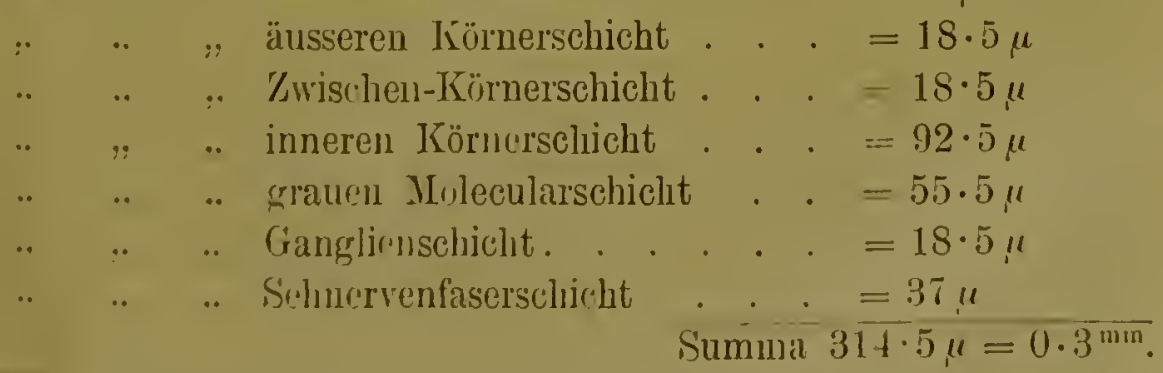

${ }^{1}$ I'apilla . . tardissirne movetur. Soemineriug, 1.c. p. 58. 
Ich konnte iu der mir zugänglichen Literatur eine ähnliche Messung der Froschretina nicht auffinden, will der meinigen auch keinen allgemeingültigen Werth beilegen; glaube aber, dass sie genügt, eine Grundlage für unsere annähernde Refractions-Berechnung abzugeben.

In Sömmering's Horizontalschnitt von Rana temporaria ist die

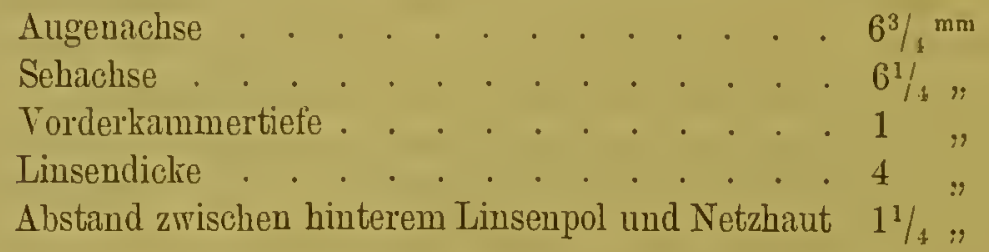

Seine Beschreibung lautet im Auszug:

Ranae temporariae bulbus oculi maximus, fere globosus, ante paullum depressus. Cornea magna, circularis, parum convexa, segmentum ejusdem sphaerae quam sclerotica efficit. Lens grandissima, fere globosa, maximum oculi spatium occupans, ita ut corneae et retinae proxima sit.

Vielleicht ist es nicht unzweckmässig, aus Sömmering's eigner Tabelle eimen Auszug, betreffend das Auge des Menschen, des Frosches und des Hechtes, beizufügen. Das Längenmaass ist die Linie.

\section{Mensurae oculorum.}

\begin{tabular}{|c|c|c|c|c|c|c|c|c|c|c|c|c|}
\hline & $\stackrel{\dot{n}}{3}$ & 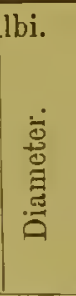 & 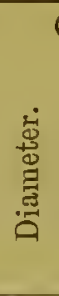 & 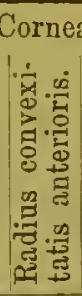 & 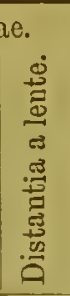 & $\frac{\dot{\theta}}{\frac{\Delta}{4}}$ & 剀 & 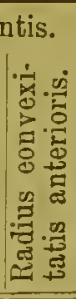 & 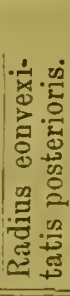 & $\cdot \vec{n}$ & 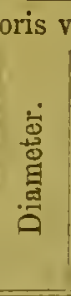 & 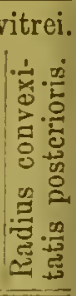 \\
\hline \multirow{3}{*}{$\begin{array}{l}\text { Puella XX annorum } \\
\text { Rana temporaria } \\
\text { Esox lucius. }\end{array}$} & $10 \cdot 0$ & $9 \cdot 5$ & $4 \cdot 5$ & $3 \cdot 3$ & $1 \cdot 3$ & $1 \cdot 6$ & $3 \cdot 6$ & $4 \cdot 2$ & $2 \cdot 4$ & $6 \cdot 2$ & $8 \cdot 7$ & $4 \cdot 4$ \\
\hline & $3 \cdot 2$ & $3 \cdot 8$ & $2 \cdot 6$ & $2 \cdot 1$ & 0.4 & $1 \cdot 6$ & $2 \cdot 0$ & $1 \cdot 1$ & $1 \cdot 0$ & 0.6 & $3 \cdot 4$ & $1 \cdot 8$ \\
\hline & $8 \cdot 5$ & $10 \cdot 5$ & $8 \cdot 5$ & $6 \cdot 0$ & 0.2 & $3 \cdot 4$ & $3 \cdot 4$ & $1 \cdot 7$ & $1 \cdot 7$ & $2 \cdot 0$ & $7 \cdot 7$ & $4 \cdot 2$ \\
\hline
\end{tabular}

Aber ist es mir denn gelungen, mit den wenigen einfachen Versuchen, wie sie die spärliche Musse des beschäftigten Praktikers zulässt, in der That die grosse Zahlenreihe zu entkräften, welche Hr. Plateau uach langem, sorgsamem, über die ganze Wirbelthierreilıe ausgedehntem Studium in seiner preisgekrönten Denkschrift niedergelegt hat? Jit Recht sagt Plateau, dass vor ihm die Literatur zwar zahlreiche anatomische Forschungen, aber in physiologischer Hinsicht mur theoretische Hrörterungen, keine directen Ter- 
ersuche über das Sehen der Amphibien und Fische enthalten habe. Und elche Versuche hat er denn selber an lebenden Auge angestellt? Er isst das Fensterkreuz ron der Hormlıaut eines lebenden Fisches spiegeln. a der Witte erscheint das Kreuz fast gerade, wälrend oben und unten die inien sich krümmen und zurücktreten: „Tandis que les portions supérieures inférieures s'incurrent et fuient". Hieraus schliesst er, dass die Krümlung in der Mitte einer Ebene sich nähert, am Rande viel stärker sei. on einer Messung der Breite der Reflexbilder ist keine Rede. Er verisst auch sofort diese Methode und giebt dem Eingypsen der herausgeommenen Augen den Vorzug. Er zeichnet die Contourlinien der Gypsnodelle und bildet sie ab. Er erhält für die Hornhautcontur bei Fischen nd Amphibien die Form eines vorn abgestumpften Kegels. Niemals findet nan den Durchschnitt gut gehärteter Amphibien- und Fischaugen vou olcher Form.

Auch in den lilassischen Abbildungen von Sömmering ist davon ichts zu entdecken, wiewohl wir auch hier den Epicyclus der Fischcornea ermissen, der ja aber nicht gerade genau in die Horizontalebene hineinufallen braucht.

Das Eingypsen giebt die Form des menschlichen Fusses unter noralen und pathologischen Verhältmissen genau wieder, aber nicht die der isch- und Froschcornea, und kaun mit der optischen Reflexmethode nicht n Genauigkeit rerglichen werden. Beim Eingypsen wurde der Epicyclus ntweder platt gedrückt oder falsch localisirt. ${ }^{1}$ Beim Hecht fand Platea u en Krümmungsradius des angeblich abgeplatteten Hornhautcentrums $=171 / 2$ is $20^{\mathrm{mm}}$, den der lateralen Hornhautpartieen $=8^{\mathrm{mm}}$, die Breite der abeplatteten Partie $4^{\mathrm{mm}}$ und die Breite der ganzen Hornhant $=13^{\mathrm{mm}}$.

Ich fand bei einem Hecht, dessen Hornhaut $12 \mathrm{~mm}$ breit war, am emporalen Pande des Pupillarbereiches auch einen Hormhantradius ron $6^{\mathrm{mm}}$, aher vor der Mitte der Pupille, und vor dem nasalen Rande einen jchen ron $12 \mathrm{~mm}$.

Celurigens musste Platea mitunter, wenigstens bei Vögeln (l. c. p. 24) inen gerrissen Druck auf den herausgenommenen Augapfel ausüben, um im die zum Nodelliren geeignete Form zu geben! Bei Rana temporaria and er in der abgeplatteten Xitte der Hornhant den Krümmungsradius ron bis $11 \mathrm{rm}$, am Rande der Hornhaut von $4 \mathrm{~mm}$, die Augenaxe $8 \mathrm{~mm}$; bei 'una esculenta den Krümmungsrarlius in der flachen Mittelpartie der Horn-

1 Platean, 1. c. Fxplication de la planche: Chaque figure représcnto la coupe rizontale, telle que la donne le moulagc; le erystallin y a été ajouté ensuite. (Sic!)

Fïr den Hecht (Fig. 1) ist dic Jinse sicher nicht richtig wieder hineingepackt ordes: der '/onularraun ist beirn lebenden Thiere nasenwärts broiter, in Pla teau's igur aber schläfenwärts. 
haut 5 bis $6 \mathrm{~mm}$, am Rande $3 \mathrm{~mm}$, die Augenaxe $5.5^{\mathrm{mm}}$. Hierzu habe ich zweierlei zu bemerken: 1) Der Spiegelversuch, namentlich mit dem Keratoskop, zeigt unwiderleglich, dass im Pupillarbereich die Hornhaut des Frosches so regelmässig gekrümmt ist wie die des Menschen, und je nach der Grösse des Auges Krümmungsiadien von $4-5^{\mathrm{mm}}$ besitzt. 2) Die Zahlen des Hru. Plateau als richtig zugegeben, wie kann man die Wirkung einer solchen Hormhaut in Luft $=0$ setzen, wenn ihre vordere Hauptbrennweite zwischen 27 und $33 \mathrm{~mm}$ nach seinen eigenen Zahlen zu berechnen wäre? Nach meinen beträgt sie zwischen 12 und $15 \mathrm{~mm}$.

$\mathrm{Hr}$. Plate a hat endlich am hinteren Pol des herausgenommenen Auges ein Lichtfenster präparirt, in dasselbe ein passendes Stück (fragment convenable) aus einer geblasenen Hohlglaskugel eingesetzt, das er zwischen Sclerotica und Glasliörper einschob, und nun die Einstellung eimes solchen Auges in Luft und in Wasser gemessen. Er fand für den Hecht die Fernpunktdistanz in Luft 40 , in Wasser $50 \mathrm{~mm}$; für den Frosch in Luft 35 , in Wasser $36 \mathrm{~mm}$. Diese Resultate sind an sich unwallrscheinlich und im Widerspruch mit der Dioptrik. ${ }^{1}$ Trotz der angewendeten Lupenvergrösserung ist die Exactheit seiner Methode nur eine scheinbare. Beim Fisch liegt die Netzhautebene beträchtlich vor der Sclerotica. Bei Sömmering messe ich die Augenare des Hechtes = $20 \mathrm{~mm}$, die Sehaxe $=15 \mathrm{~mm}$. Differenz $5 \mathrm{~mm}$ oder $1 /$ der Sehaxenlänge. Beim Menschen beträgt dieselbe Differenz nur $1 / 2 \varphi^{2}$ Plateau's k ünstliche Netzhaut liegt beträchtlich hinter der natürlichen; das künstliche Auge muss auch in Wasser excessivkurzsichtig sein. - Beim

1 Selbst wenn wir dem Hechtange unter Wasser eine so excessive Myopie geben wollten, dass die Hinzufügung eines brechenden Hornhautsystems ron $20 \mathrm{~mm}$ Radius (Plateau's Maximum), also $60 \mathrm{~mm}$ vorderer Brennweite keine erhebliche Verrückung des Fernpunktes einführte, würden wir die obigen Zahlen nicht erhalten können. $\frac{1}{50}+\frac{1}{60}=\frac{110}{3000}=\frac{1}{27}$. Wäre die Fernpunktsdistanz des Hechtauges in Wasser $50 \mathrm{~mm}$, so müsste sie in Luft $27 \mathrm{~mm}$ betragen. $\frac{1}{40}-\frac{1}{60}=\frac{20}{2400}=\frac{1}{120}$. Wäre die Fernpunktsdistanz desselben in Luft $40 \mathrm{~mm}$, so würde sie in Wasser $120 \mathrm{~mm}$ ausmachen. Vollends beim Frosch, wo $r$ nach Platea u $10 \mathrm{~mm}$ max. betragen soll.

$\frac{1}{36}+\frac{1}{30}=\frac{1}{15}$. Wäre die Fernpunktdistanz des Froschauges in Wasscr 36 mm, so wiurde sie in Luft $15 \mathrm{~mm}$ betragen.

$\frac{1}{35}-\frac{1}{30}=-\frac{1}{210}$. Wäre die Fcrnpunktdistanz derselben in Isuft $+35 \mathrm{~mm}$, so müsste sie unter Wasser $-210 \mathrm{~mm}$ bctragcn.

${ }^{2}$ Meine eigene Messung ergab fïr den Hecht:

Augenaxe $s=15 \mathrm{~mm}$ ।

$\left.\begin{array}{ll}\text { Augenaxe } & s=15 \mathrm{~mm} \\ \text { Sehaxe } & \sigma=12 \mathrm{~mm}\end{array}\right\} s-\sigma=\frac{1}{3} s$. 
Die Blutgefässe, welche aus dem Mittelpunlit des Sehnerveneintritts hervorkommen und die ganze Netzhaut, soweit dieselbe sichtbar, überzichell, sind im ganzen radiär angeordnet, dichotomisch verästelt, mit ceutralem Reflexstreif wie in der menschlichen Netzhaut versehen. Bei den Hauptästell kann man, wie es scheint, die arteriellen von den venösen unter-? scheiden, die ersteren sind etwas schmäler und heller roth als die letzteren.

Das Centrum der weissen Sehnervenscheibe, der Sammelpunlit der convergirenden Blutgefässe, ist dunkelgrauroth gefärbt.

Bei grösseren Plötzen sieht man deutlich, dass 1) das rundliche Centrum der weissen Sehnervenscheibe an sich dunkelgrau gefärbt und nur mit verhältnissmässig breiten und zahlreichen rothen Streifen, den Blutgefässen, belegt ist; und dass 2) einzelne von den auch in unserer Figur angedeuteten Blutgefässbögen in den Glaskörperraum hineinragen.

Das Augengrundsbild des Aales gestaltet sich nach Fig. 6. (Aufrechtes Netzhautbild des rechten Auges von einem kleinen Aal; nach dem umge-

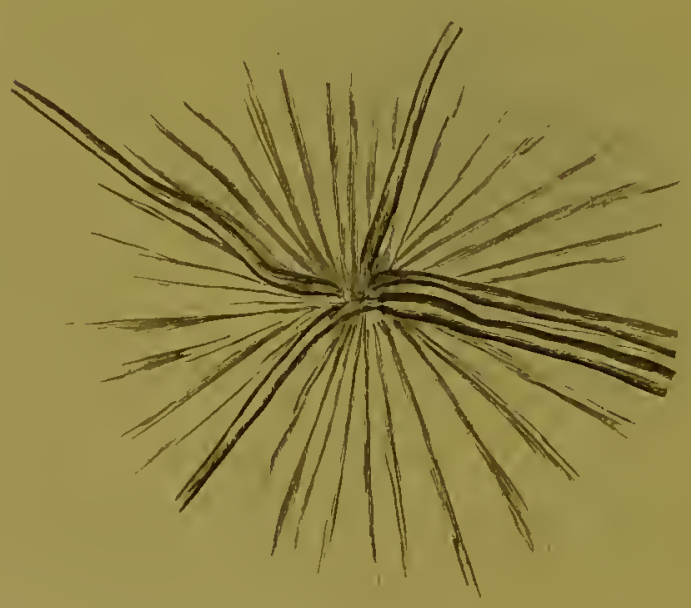

Fig. 6. kehrten, mit einer Linse von $2^{\prime \prime}$ Bremnweite gewonnenen vergrössert) folgendermaassen:

Eine eigentliche Papille ist nicht vorhanden, sondern nur der Mittelpunkt der retinalen Sehmerrenfaserausstrahlung nachweisbar.

Von diesem Mittelpunlitaus ziehen kräftig gezeichnete schmale, weisshiche Sehnerrenfaserbündel, radiär angeordnet, ziemlich weit hinein in die Netzhaut, im Ganzen etwa 2-3 Dutzend, so dass zwischen je zwei Bündeln ein Winkel von ungeführ $10^{\circ}$ bleibt.

Aal. Rechtes Auge. Aufrechtes Netzhautbild.

Der Convergenzpunkt der Sehnerrenfaserbündel fällt zusammen mitdem Confluenzpunkt der retinalen Blutgefässe. Die letzteren ziehen etria nach vier diagonalen Richtungen, zwei kiemenwärts, drei nach der entgegengesetzten Richtung. Wie es scheint sind drei renöse und zwei etwas feinere und heller rothe arterielle Aeste rorhanden, doch ist das Bild nicht deutlich genug, um dieses zu entscheiden. Die Blutgefässe sind roth, mit centralem Reflexstreif, wie auf der menschlichen Netzhaut.

Das Bild des linken Auges, namentlich auch die Vascularisation, war ebenso wie das des rechten: Zwei starke Gefïsse ziehen dicht nebun ein- 
ander ron innen unten her llach dem anatomischen Vittelpount der Netzhaut.

Das Augengrundsbild des Hechtes (rgl. Fig. 7) weicht ron dem der. bisher betrachteten Fische erheblich ab.

Blickt man nach aussen unten in das Hechtange, so sieht man einen eigenthümlichen, sehr langen, weissen, fast wurmähulichen, von aussen oben nach innen unten gerichteten Streifen, der sich deutlich in drei $A b$ sthnitte gliedert.

Der oberste Theil des, wie es scheint, cylindrisch hervorragenden Streifens begiunt mit einer knopfförmigen Anchwellung, ist parallelrandig und beiderseits mit einem schmalen Pigmentsaum versehen. Der mittlere Theil ist bauchig angeschwollen und trägt cine ähulich gestaltete schwarze Pigmentinsel. Der unterste Theil ist dünn, fast fadenformig und sein unteres Ende im Augenspiegelbilde nicht zu erreichen.

Nur ron dem mittleren Theil scheinen die Sehnerrenfaserm auszustrahlen und zwar mehr nach immen als nach ausseu. In der inneren Hälfte der Sehnervenfaserausstrahlung heben sich zwei kräftige Bündel besonders ab, welche ungefähr ron dem oberen und von dem unteren Ende des bauchigen Theiles rom Papillarstreifen auszugehen scheinen.

Der ganze sichtbare Augengrund ist vollkommen gefässlos und röthlich, soweit nicht die Sehnertenfaserausstrahlung einen reisslichen oder weissgrünlichen Farbentou darüber legt. Bei anderen Exemplaren war temporalwärts

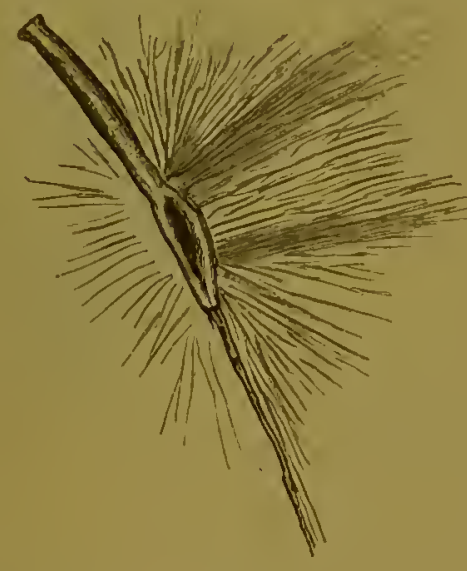

Fig. 7.

Aufrechtes Bild des rechton Auges vom Hecht. überhaupt keine Sehnervenfaserung zu entdecken; am obersten Theil des Streifens fehlte der Pigmentsaum zu einer Seite, am mittleren Theil war die Pigmentinsel mehr nach der einen Seite verlagert.

Mit dieser Schilderung des Augenspiegelbefundes, die ich vor jeder eigenen anatomischen Tntersuchung entworfen, wolle man die oben schon mitgetheilte Beschreibung von Sömmering vergleichen.

Das Augengrundshild des Frosches ist genügend bekannt und auch von mir selber schon ausführlich geschildert. Für die Entwerfung der beifolgenden schematischen Zeichnung (Fig. 8) wurde ein Exemplar bellutzt, bei dem die Art. hyaloides ziemlich wahe der Vena verlief. 1)ie glitzernden Pankte des Augengrundes wurden fortgelassen. Das Capillarnetz ist nur thrilweise wiedergegeben. 
p Papille.

$v$ Venen.

$\checkmark$ Vena hyaloides (Hauptstamm).

a Arteria hyaloides.

$C_{1}$ Capillarnetz, in welches die Arterie sich auflöst.

$C_{2}$ Capillarnetz, welches die Vene zusammensetzt.

$C_{3}$ Capillarnetz, welches in gröbere Venenäste hineiumündet. Die Pfeile bedeuten die Stromesrichtung.

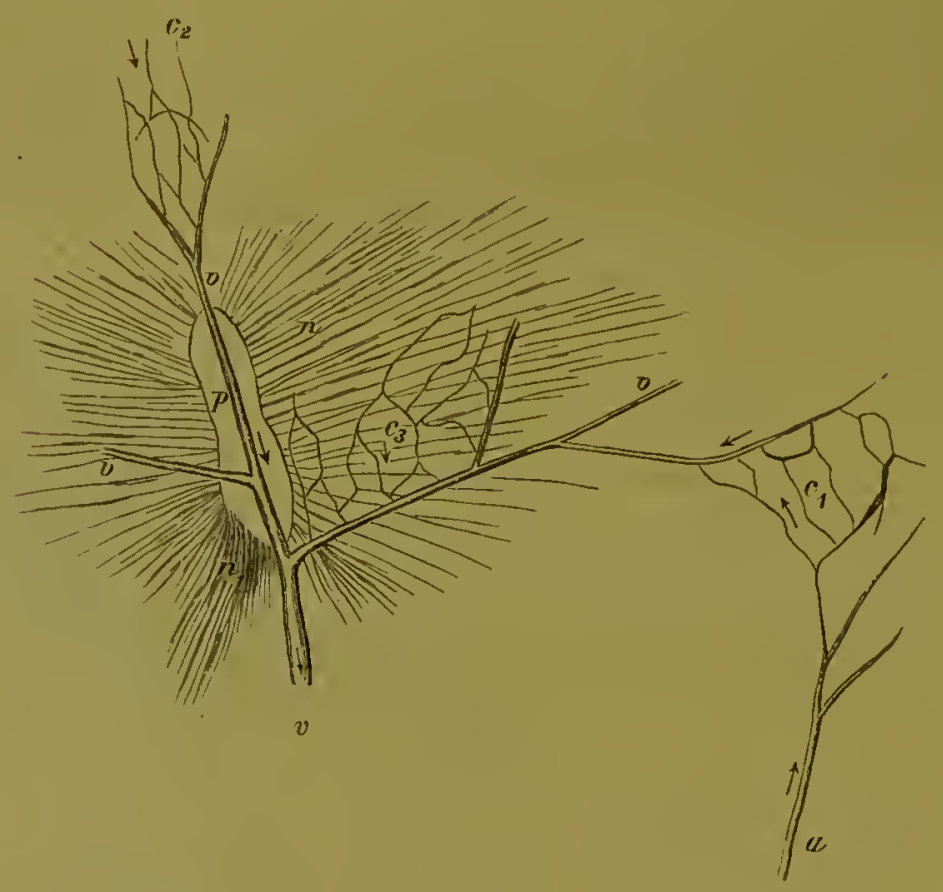

Fig. 8.

Aufrechtes Bild des rechten Auges vom Frosch.

\section{Anhang. Elementare Dioptrik der Kugellinsen."}

$O$ sei ein beliebiger Axenpunkt, von welchem ein beliebiger Strahl $O J$ auf die kugelige Treunungsfläche $B_{1} B_{2}$ (etwa zwischen dem Medium

1 Seitdem man nicht mehr geschmolzene Kügclehen zu eivfachen Mikroskopen verwendet, ist dieses Capitel seheinbal ohne praktischen Werth und deshalb in den neuen Lehrbïchern stiefmütterlich behandelt. Die Dioptrik von Mathicssen (I,eipzig 1877. S. 118) hat 5, die von Fcriaris-Lippieh (Jeipzig 1879. S. 90) 7 Zeilen über diesen Gegenstand. Der alte Klügel (Leipzig 1778) ist ausführlich, aber nicht ganz bequem für unsere Zwecke. 
$\mathrm{I}=$ Luft und den Melium $\mathrm{II}=$ Glas) füllt. Der Radius $M . J K$ ist das Einfallsloth: $\angle O J K=\angle J$ ist der Einfallswinkel, $\angle M J P=0$ der Brechungswinkel. Wenn alle Strahlen nahezu senkrecht auf $B_{1} B_{2}$ ein-

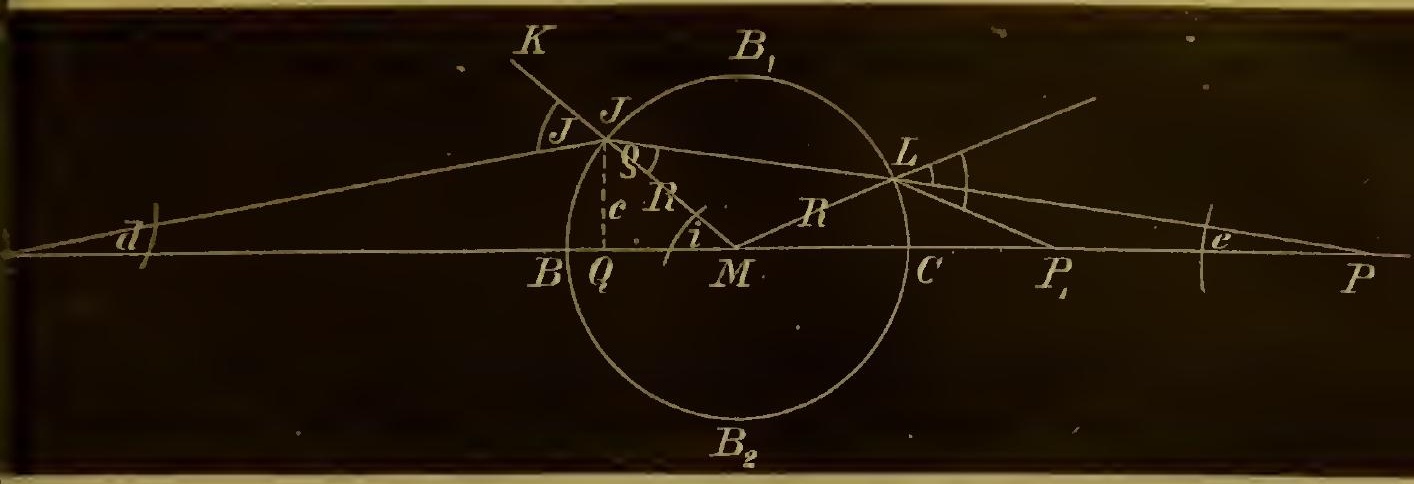

Fig. 9.

fallen, d. h. alle Einfallswinkel so klein sind, dass sie gleich ihren Sinus uder Tangenten gesetzt werden können (d. h. $Q_{1}$ der Fusspunkt des Lothes $J Q=c$, sehr nahe an $B$ fällt), so ist das Brechungsgesetz

1) $n_{1} J=n_{2} \varrho$ oder $\left.J=\frac{n_{2}}{n_{1}} \varrho ; 1^{a}\right) \varrho=\frac{n_{1}}{n_{2}} J$. Ferner ist immer

2) $J=d+i$

3) $\quad i=\varrho+e$ oder $\left.3^{a}\right) \varrho=i-e$.

Wird in $1^{a}$ für $\varrho$ der Werth von $3^{a}$, und für $J$ von 2 eingesetzt, so folgt

$$
\begin{aligned}
i-e=\frac{n_{1}}{n_{2}}(d+i) & \text { oder } \frac{n_{2}}{n_{1}}(i-e)=d+i \\
\text { oder } n_{2}\left(\frac{c}{R_{1}} \cdot-\frac{c}{f_{2}}\right) & =n_{1} \frac{c}{R_{1}}+n_{1} \frac{c}{f_{1}},
\end{aligned}
$$

wenn $O B=O Q=f_{1}$ und $B P=Q P=f_{2}$ gesetzt wird. $R_{1}$ ist der Krümmungsradius der ersten Kugelfläche; $c$ hebt sich fort: es bleibt

$$
\begin{aligned}
& \frac{n_{2}}{R_{1}}-\frac{n_{1}}{R_{1}}=\frac{n_{2}}{f_{2}}+\frac{n_{1}}{f_{1}} \text { orler } \\
& \text { 1. } \quad \frac{n_{2}-n_{1}}{k_{1}}=\frac{n_{1}}{f_{1}}+\frac{n_{2}}{f_{2}} .
\end{aligned}
$$

Diese Gleichung bestimmat eindentig den Vereinigungspunct des schmalen, won () divergirenden Strahlenbündels. Ist das System $\left(R_{1}, n_{1}, n_{2}\right)$ bekannt und die Objectferne $f_{1}$ gegeben, so wird aus Gleichung $I$ die Bildferne $f_{2}$ gefunden. Ist speciell $f_{1}=\infty$, so wird der dazu gehörige Werth von $f_{2}$, den wir $F_{2}$ nemen wollen, gegeben durch

$$
\frac{n_{2}-n_{1}}{K_{1}}=\frac{n_{2}}{F_{2}} \quad \text { oder II. } \quad F_{2}=\frac{n_{2} R_{1}}{n_{2}-n_{1}} \text {. }
$$


Ist $f_{2}=\propto$, so ist der dazu gehörige Werth ron $f_{1}$, den wir $F_{1}$ nennen wollen,

$$
\text { III. } \quad F_{1}=\frac{n_{1} R_{1}}{n_{2}-n_{1}}
$$

$F_{1}$ und $F_{2}$ sind die Hauptbrennweiten des einfachen systems.

Folgt auf der nämlichen Axe bald hinter $B_{1} B B_{2}$ noch eine zweite Krugelfläche $B_{1} C B_{2}$ zwischen Medium II und III (III lann wieder Luft sein), so liömnell wir die Formel I sofort auf die Brechung an dieser zweitell Fläche anwenden. Wir haben aber zu berücksichtigen, dass die Concavität dieser Kugelläche dem einfallenden Strahl zugewendet ist und deshalb der Radius $R_{2}$ derselben negativ zu nehmen und statt $n_{2}$ immer $n_{1}$ und statt $n_{1}$ immer $n_{2} z u$ setzen ist. Denn das erste Medium für die zweite Brechung setzen wir identisch dem zweiten Medium für die erste Brechung. Das dritte Medium sei identisch mit dem ersten. Es sei $P_{1}$ der definitive Bildpunlit und seine Entfernung ron der zweiten KugelHäche $P_{1} C=\varphi_{2}$. Die Entfernung des Objectpunlites für die zweite Brechung $(P)$ vou der zweiten Kugelfläche ist $P C=-\varphi_{1}$, negatir zu setzen, weil der Objectpunlit im Gange der Lichtstrahlen hinter der brechenden Fläche $\left(B_{1} C B_{2}\right)$ belegen ist.

Dann gilt also laut Gl. I:

$$
\text { 1) } \frac{1}{\tau_{2}}=\frac{1}{n_{1}}\left\{\frac{n_{1}-n_{2}}{-R_{2}}-\frac{n_{2}}{-r_{1}}\right\} \text { oder } \frac{1}{r_{2}}=\frac{1}{n_{1}}\left\{\frac{\left(n_{2}-n_{1}\right)}{R_{2}}+\frac{n_{2}}{\tau_{1}}\right\}
$$

Nun ist 2) $B P-B C=C P$

oder $f_{2}-\left[R_{1}+R_{2}\right]=\varphi_{1}$. Uebrigens ist mach der Toraussetzung einer Kugellinse $R_{1}=R_{2}$ in absolutem Maasse; also

$$
\text { 3) } \frac{1}{r_{2}}=\frac{1}{n_{1}}\left[\frac{n_{2}-n_{1}}{R}+\frac{n_{2}}{f_{2}-2 R}\right]
$$

Wir suchen zunächst nur den Vereinigungspunlit parallel auf die erste Kugelfläche fallender Strahlenbündel, d. h. wir setzen $f_{1}=\infty_{1}$, dann wird speciell $f_{2}=\frac{n_{2} R}{n_{2}-n_{1}}:$ also

$$
\text { 4) } \begin{aligned}
\frac{1}{r_{2}} & =\frac{1}{n_{1}}\left[\frac{n_{2}-n_{1}}{R}+\frac{n_{2}}{\frac{n_{2} R}{n_{2}-n_{1}}-2 R}\right] \\
\frac{1}{r_{2}} & =\frac{1}{n_{1}}\left[\frac{\left(n_{2}-n_{1}\right)}{R}+\frac{n_{2}\left(n_{2}-n_{1}\right)}{n_{2} R-2 R\left(n_{2}-n_{1}\right)}\right] \\
1 & =\left(\begin{array}{c}
n_{2}-n_{1} \\
n_{1}
\end{array}\right)\left[\begin{array}{c}
n_{2} R-2 R\left(n_{2}-n_{1}\right)+n_{2} R \\
R\left(n_{2} R-2 R\left[n_{2}-n_{1}\right)\right.
\end{array}\right]
\end{aligned}
$$




$$
\begin{aligned}
\frac{1}{r_{2}} & =\frac{u_{2}-n_{1}}{n_{1}}\left[\frac{2 n_{3}-2\left(u_{3}-u_{1}\right)}{u_{2} R-2 R\left(n_{2}-n_{1}\right)}\right] \\
\frac{1}{r_{2}} & =\frac{2\left(n_{2}-n_{1}\right)}{n_{1} R}\left[\frac{n_{2}-n_{2}+n_{1}}{n_{2}-2 u_{3}+2 n_{1}}\right] \\
1 & =\frac{2\left(n_{2}-u_{1}\right)}{n_{1} R} \times\left(2 n_{1}\right. \\
\psi_{2} & =\frac{\left(2 n_{1}-n_{2}\right)}{2\left(n_{2}-n_{2}\right) R} .
\end{aligned}
$$

$\varphi$ : giebt den Abstand des hinteren Brenupunktes der Kugellinse von der Hinterfläche derselben. Es ist klar, dass der optische Mittelpunkt der Kingellinse (der Ĺnotenpunkt) mit dem geometrischen zusammenfällt. Denn ein durch letateren zielender Strahl fällt lothrecht auf beide Kugelflächen und wird nicht abgelenkt.

Beispiel 1) Eine Glaskugel sei in Luft getaucht: $n_{1}=1 ; n_{2}=\frac{3}{2}$.

$$
\mathscr{f}_{2}=\frac{2-\frac{3}{2}}{2\left(\frac{3}{2}-1\right)} R=\frac{\frac{1}{2} R}{2 \times \frac{1}{2}}=\frac{1}{2} R \text {. }
$$

Der Vereinigungspunkt des parallel der Hauptaxe auf die gläserne, in Luft befindliche Kugellinse fallenden Strahlenbündels liegt um die Hälfte des Radius hinter dem hinteren Pol der Kugel.

2) Eine Wasserkugel sei in Luft getaucht: $n_{1}=1, \mathrm{n}_{3}=\frac{4}{3}$.

$$
f_{2}=\frac{\left(2-\frac{1}{3}\right) R}{2\left(\frac{1}{3}-1\right)}=\frac{\frac{2}{3} R}{\frac{2}{3}}=R \text {. }
$$

Der Tereinigungspunkt liegt um die Länge des Radius hinter dem hinteren Pol der Kugel.

3) Eine Glaskugel sei in Wasser getaucht: $n_{1}=\frac{1}{3} ; n_{2}=\frac{3}{2}$

$$
\begin{aligned}
\mathscr{w}_{2} & =\frac{\left(2 \cdot \frac{1}{3}-\frac{3}{2}\right) R .}{2\left(\frac{3}{2}-\frac{1}{3}\right)}=\frac{\left(\frac{8}{3}-\frac{3}{2}\right) R}{\frac{2}{6}}=\frac{\frac{16}{6}-\frac{9}{6}}{\frac{2}{6}} R \\
& =\left(\frac{16-9}{2}\right) R=\frac{7}{2} R=3 . \frac{2}{6} R .
\end{aligned}
$$

Vennen wir die Brennweite der Kugellinse (Abstand des Brenn- vom Gnotenpunkt) $\mathscr{L}_{1}$; so ist dieselbe für die drei Beispiele:

1) $\|_{1}=\Omega_{i}+\frac{R}{2}=1 ! R$

2) ()$_{2}=R+l i=2 \pi$

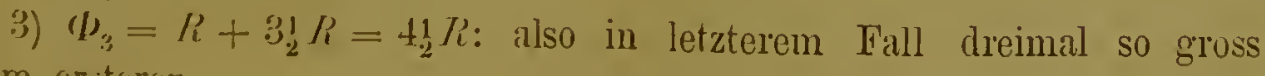
Ils im ersteren.

Ian könnte geneigt sein, den letateren Fall auf die Krystallinse des elenden in Wasser getauchten Fisches anzuwenden: dann wären die Fisch- 
augen stark übersichtig in Wasser. Denn der Abstand der Netzhaut ron der hinteren Linsenfläche ist betrïchtlich kleiner als $3 \frac{1}{2} R$.

Matthiessen (Dioptrık S. 218) fand beim Seebarsch den Brechungsindex :

$$
\begin{array}{ll}
\text { der Rinde . . } & 1.43 \\
\text { der Mittel-Schicht . } & 1.49 \\
\text { des Kernes . . . } & 1.51
\end{array}
$$

und berechnet daraus den totalen Brechungsindex $n=1.7$ und die Brennweite $=5 \cdot 5$. Der Durchmesser der Linse war 5.0; Krümmungsradius del Hornhaut 6.6 ; Ort der vorderen Linsenfläche 0.5 ; Ort der Retina 8.5 . Also läge die Retina $3 \mathrm{~mm}$ hinter der Hinterfläche der Linse und der Vereinigungspunkt eines parallel einfallenden Strahlenbündelss nahezu in der Retina.

Setzen wir

$$
n_{2}=1 \cdot 7 \quad n_{1}=1 \cdot 3
$$

so folgt $\varphi_{2}=\frac{(2 \times 1.3-1 \cdot 7)}{2(1 \cdot 7-1 \cdot 3)} 2 \cdot 5=\frac{(2 \cdot 6-1 \cdot 7)}{0.8} \times 2.5=\frac{0.9}{0.8} \times 2 \cdot 5$

d. h. fast in der Retina. Danach wäre auch

$$
\Phi=2 \cdot 5+2 \cdot 75=5 \cdot 25
$$

was genügend mit Matthiessen's Integralrechnung übereinstimmt.

Der totale Brechungsindex der Fischlinsen ist grösser als der des Glases.

Natürlich gilt auch für Kugellinsen:

$$
\frac{\beta_{1}}{-\beta_{2}}=\frac{r_{1}}{F_{1}}=\frac{F_{2}}{p_{2}}
$$

